\title{
Übergangslösungen beim Eintritt in die Schweizer Berufsbildung: Brückenschlag oder Sackgasse?
}

\author{
Stefan Sacchi* und Thomas Meyer* \\ *TREE, Universität Bern, CH-3012 Bern, stefan.sacchi@soz.unibe.ch und thomas.meyer@soz.unibe.ch.
}

\section{Abstracts \\ Übergangslösungen beim Eintritt in die Schweizer Berufsbildung: Brückenschlag oder Sackgasse?}

Am Übergang in nachobligatorische Ausbildungsgänge durchlaufen viele Jugendliche in der Schweiz eine sogenannte Übergangslösung oder ein Brückenangebot wie z. B. ein zehntes Schuljahr. Wir beleuchten in diesem Beitrag zum einen, wie schulische, individuelle, familiäre und systemische Kontextfaktoren den Übertritt in solche Brückenangebote beeinflussen. Zum anderen gehen wir der Frage nach, wie sich ein verzögerter Einstieg über ein Brückenangebot auf die Chance auswirkt, eine Ausbildung auf Sekundarstufe II zu beginnen bzw. abzuschliessen. Auf der Basis der TREEDaten modellieren wir zunächst die interessierenden Übertrittsprozesse mittels einer multinomialen logistischen Regression, um dann mittels Propensity Score Matching deren Wirkung auf die nachobligatorischen Bildungschancen abzuschätzen.

Schlüsselwörter: Brückenangebote, Transitionen zwischen der Sekundarstufe I und der Sekundarstufe II, nachobligatorische Ausbildungsverläufe, Dropout, Bildungsungleichheit

\section{Entrée en formation professionnelle par des solutions transitoires: passerelle ou impasse?}

En Suisse, beaucoup de jeunes passent à une formation post-obligatoire par l'intermédiaire de solutions transitoires comme une $10^{\text {ème }}$ année scolaire. Dans cet article, nous analysons d'abord comment les facteurs contextuels individuels, familiaux, scolaires et systémiques influencent le passage à une solution transitoire. Ensuite, nous examinerons les effets du recours à une solution transitoire sur la chance d'accéder à une formation de niveau secondaire II et de l'achever. L'analyse s'appuie sur les données de l'enquête TREE. Au moyen d'une régression logistique multinomiale, nous modélisons d'abord les processus de sélection. Ensuite, nous estimons les effets de ces processus sur la probabilité de réussite du parcours de formation post-obligatoire au moyen d'un modèle d'appariement par score de propension.

Mots-clés: Solutions transitoires, passage du secondaire I au secondaire II, parcours de formation post-obligatoires, dropout, inégalité de la formation

\section{VET Entry in Switzerland Via "Bridging Solutions": Stepping Stone or Dead End?}

During transition to post-compulsory education, a considerable proportion of Swiss school leavers pursues so called intermediate or bridging solutions, usually one-year programs which are geared to facilitate access to VET. In this contribution, we analyze how individual, family, school and systemic context factors influence such a delayed transition, and which effect this transition has on upper secondary VET enrolment and completion. On the basis of the TREE data we first model the selection processes at work by means of a multinomial logistic regression. Then we estimate the effects on access to and completion of an upper secondary VET program by means of propensity score matching.

Keywords: Bridging solutions, transition from lower to upper secondary education, post-compulsory education pathways, early dropout, educational inequality 


\title{
Übergangslösungen beim Eintritt in die Schweizer Berufsbildung: Brückenschlag oder Sackgasse?
}

\author{
Stefan Sacchi* und Thomas Meyer *
}

\section{$1 \quad$ Einleitung}

In modernen wissensbasierten Gesellschaften ist ein Bildungsabschluss der Sekundarstufe II - in der Folge auch kurz als Sek.-II-Abschluss bezeichnet - ein qualifikatorischer Mindeststandard, der eine Integration ins Berufsleben und volle gesellschaftliche Teilhabe ermöglicht (Solga 2005). In der Schweiz erlangen heute rund neun von zehn Jugendlichen einen entsprechenden nachobligatorischen Abschluss (Schweizerische Koordinationsstelle für Bildungsforschung 2014, 110 f.). Auch die Schweizer Bildungspolitik erachtet heute einen Sek.-II-Abschluss als Mindeststandard, den möglichst viele Jugendliche erreichen sollen. Die Schweizerische Konferenz der kantonalen Erziehungsdirektoren (EDK) hat als Zielvorgabe definiert, dass die Sek.-II-Abschlussquote bis 201595 Prozent erreichen solle (Schweizerische Koordinationsstelle für Bildungsforschung 2014).

Die Schulpflicht endet in der Schweiz mit dem neunten Schuljahr, nachdem die Jugendlichen die Sekundarstufe I durchlaufen haben. Auf der Sekundarstufe II besteht weder eine formelle Verpflichtung zur Fortführung der Ausbildung noch ein gesetzlicher Anspruch auf einen Ausbildungsplatz. Die Erfüllung der Ausbildungsnorm eines Sek.-II-Abschlusses liegt somit in der individuellen Verantwortung der Jugendlichen und ihres Umfelds.

Viele der aus der Schulpflicht entlassenen Jugendlichen stossen allerdings bereits beim Übertritt in die Sekundarstufe II auf Schwierigkeiten. Davon betroffen sind hauptsächlich Schulentlassene, die eine berufliche Grundbildung ${ }^{1}$ anstreben, die in der Schweiz vorwiegend in Form von dualen Berufslehren angeboten werden. Der beruflichen Grundbildung sind über 70 Prozent aller Sek.-II-Abschlüsse zuzurechnen. Rund einem Drittel der Schulentlassenen, die eine berufliche Grundbildung anstreben, bleibt der direkte Übergang in diese - meist nach einer erfolglosen Lehrstellensuche - zunächst verwehrt (Keller et al. 2010, 7; Meyer und Bertschy 2011; ähnlich für Deutschland: Beicht 2009, 4).

TREE, Universität Bern, CH-3012 Bern, stefan.sacchi@soz.unibe.ch und thomas.meyer@soz. unibe.ch.

1 Dazu zählen wir sämtliche dualen oder vollzeitschulischen Ausbildungen, die in den Erwerb eines Eidgenössischen Fähigkeitszeugnisses (EFZ) in einem der rund 250 Lehrberufe in der Schweiz münden. 
Eine überwiegende Mehrheit der Schulentlassenen, die nicht direkt in eine Sek.-II-Ausbildung einsteigen können, absolviert verschiedene Übergangsmassnahmen bzw. Zwischenlösungen (Hupka-Brunner et al. 2011, 176; Bayard 2011, 100). ${ }^{2}$ In der Regel handelt es sich dabei um einjährige, vorwiegend schulische Brückenangebote wie 10. Schuljahre, welche den anschliessenden Einstieg in eine zertifizierende Berufsausbildung ermöglichen sollen. Als weitere, weniger institutionalisierte Formen von Zwischenlösungen gelten auch Sprachaufenthalte, Au-Pair-Einsätze und Praktika sowie Vorkurse für zertifizierende Sek.-II-Ausbildungen mit besonderen Voraussetzungen, Vorlehren und Motivationssemester (vgl. Meyer 2003). Laut «Lehrstellenbarometer», mit dem das Staatssekretariat für Bildung, Forschung und Innovation (SBFI) die Entwicklung des Lehrstellenmarktes beobachtet, befanden sich in den Nullerjahren zeitweise fast 25000 junge Menschen in Übergangslösungen (zuletzt: SBFI 2014, 73), was nicht ganz einem Drittel eines Jahrgangs entspricht. ${ }^{3}$

Im bildungspolitischen und z. T. auch im wissenschaftlichen Diskurs wird der hohe Anteil von Brückenangeboten oft mit unzureichenden Kompetenzen oder individuellen Defiziten der betroffenen Jugendlichen erklärt, die sich bei der Suche nach einer Lehrstelle oder einem Ausbildungsplatz als hinderlich erweisen. Entsprechend seien die Gründe für den Eintritt in ein Brückenangebot in einer fehlenden "Ausbildungsreife» sowie in individuellen Defiziten hinsichtlich schulischer, sprachlicher, integrationsbezogener sowie arbeitsmarktrelevanter Kompetenzen und «Tugenden» zu suchen (BBT 2000; Egger, Dreher \& Partner 2007).

Eine weniger individualisierende Sichtweise auf die "Warteschlange» beim Übertritt in nachobligatorische Ausbildungen hebt dagegen das strukturelle Ungleichgewicht zwischen Ausbildungsplatz-Angebot und -Nachfrage hervor. Aufgrund der dominierenden Stellung der dualen Berufslehre innerhalb der schweizerischen Sekundarstufe II ist dafür vor allem die Entwicklung des Lehrstellenmarkts entscheidend. Dieser war während fast zweier Jahrzehnte durch eine anhaltende Lehrstellenknappheit gekennzeichnet, weshalb mit vielfältigen Massnahmen versucht wurde, zur Schaffung zusätzlicher Lehrstellen anzuregen (Meyrat 2004).

Der vorliegende Beitrag fokussiert vor dem soweit skizzierten Hintergrund auf zwei bildungspolitisch hochrelevante, empirisch aber noch wenig bis gar nicht untersuchte Fragen:

1. Welche individuellen und kontextuellen Faktoren tragen dazu bei, dass manche Jugendliche direkt in eine zertifizierende Ausbildung der Sekundarstufe II

2 Wir verwenden hier die Begriffe Übergangsmassnahmen und Brückenangebote synonym und fassen darunter alle Formen von schulischen und nicht-schulischen "Zwischenlösungen", mit denen im Falle eines verzögerten Eintritts in eine Sek.-II-Ausbildung die Zeitspanne bis zum Ausbildungsbeginn überbrückt wird.

3 Seit 2010 ist die Zahl Jugendlicher in der sogenannten "Warteschlange» auf unter $15000 \mathrm{zu}$ rückgegangen (SBFI 2014). Die vom Bundesamt für Statistik ausgewiesene Quote der «Übergangsausbildungen» (gemäss den aktuellsten Zahlen von 2012 rund 13\% eines Altersjahrgangs) erfasst nur einen Teil der Brückenangebote gemäss der hier verwendeten Definition. 
einsteigen können, während andere auf Brückenangebote ausweichen (müssen) oder vorzeitig aus dem Bildungssystem ausscheiden?

2. Wie wirkt sich bei gegebenen individuellen, familiären und schulischen Voraussetzungen der Umweg über ein Brückenangebot auf die Chance aus, in eine Ausbildung auf Sekundarstufe II einzutreten und diese dann auch erfolgreich abzuschliessen?

Der hier betrachtete Übergang im individuellen Bildungsverlauf entzieht sich dabei ein Stück weit den Kategorien der derzeit verbreiteten allgemeinen Erklärungsmodelle des Bildungserfolgs respektive der Bildungsreproduktion in der Tradition von Bourdieu oder Boudon (dazu Solga und Becker 2012). Einmal stellt das Durchlaufen eines Brückenangebots per se kein eigenständiges Bildungsziel dar, das zu einem definierten Abschluss führt und dessen Wert die Akteure in einer entscheidungstheoretischen Sichtweise ohne Weiteres einschätzen und gegen andere Bildungsangebote abwägen könnten. Der Übertritt in ein Brückenangebot ist zudem meist auch nicht ein proaktiv aufgrund von Nutzenabwägungen und verfügbaren familiären Ressourcen erstrebtes Ziel als vielmehr die unfreiwillige Konsequenz einer vergeblichen Lehrstellensuche. Aus diesen Gründen setzen wir mit unseren theoretischen Überlegungen (Abschnitt 2) näher beim Untersuchungsgegenstand an, indem wir auf Meyers (2003) Konzept der verschiedenen Funktionen von Brückenangeboten zurückgreifen.

Bei den empirischen Analysen zu beiden Fragestellungen fokussieren wir hauptsächlich auf Schulentlassene, die nach der obligatorischen Schule entweder in eine zertifizierende Berufsausbildung (ohne Gymnasium und Lehrkräfteseminare) oder in ein Brückenangebot übertreten. Als Kontrastgruppe ziehen wir zudem auch Schulentlassene heran, die ganz aus dem Bildungssystem ausscheiden. Dies ermöglicht uns, empirisch zwischen der Wirkung eines nicht gelingenden Direkteinstiegs und der Wirkung des Durchlaufens von Brückenangeboten zu unterscheiden.

\section{Theoretische Überlegungen und Forschungsstand}

\subsection{Hauptfunktionen von Brückenangeboten}

In der Schweiz erfüllen Brückenangebote an der Schnittstelle zwischen den Sekundarstufen I und II ähnliche Funktionen wie die sogenannten Übergangsmassnahmen im deutschen Bildungssystem. Meyer (2003) unterscheidet diesbezüglich drei Hauptfunktionen:

1. Die bereits in der Einleitung angetönte Kompensationsfunktion zielt auf eine Behebung individueller Defizite der Pflichtschulabgängerinnen und -abgänger, dank der diese erst «berufsbildungsfähig» werden. Solche Defizite werden zum einen bei den schulischen und/oder sprachlichen Leistungen und Fertigkeiten unterstellt (z. T. auch bei Techniken des «Selbst-Marketings» wie Bewerbun- 
gen schreiben, Vorstellungsgespräche führen), zum anderen bei sogenannten Arbeitsmarkttugenden (Pünktlichkeit, Zuverlässigkeit, Höflichkeit, äussere Erscheinung, usw.).

2. Mit Blick auf die Orientierungs- bzw. Berufswahlfunktion sollen Brückenangebote dabei helfen, sich über die eigenen Berufsziele und Ausbildungswünsche klar zu werden. Mitunter wird in diesem Zusammenhang auch von «Ausbildungsreife» oder "Berufsreife» gesprochen.

3. Die systemische Pufferfunktion kommt zum Tragen, wenn die Zahl der Jugendlichen, die nach dem Ende der Schulzeit eine berufliche Grundbildung anstreben, das Angebot an entsprechenden Ausbildungsplätzen übersteigt. Brückenangebote dienen dann als Auffangbecken für jene, denen der Direkteinstieg in eine zertifizierende berufliche Grundbildung nicht gelingt.

Im häufig stark individualisierenden bildungspolitischen Diskurs stehen die erste und die zweite Funktion im Vordergrund. Die dritte Funktion wurde von den Akteuren der Berufsbildungspolitik lange ignoriert. So definiert das zuständige Staatssekretariat für Bildung, Forschung und Innovation (SBFI, vormals BBT) das Zielpublikum von Brückenangeboten (BBT 2000, 4, 7) wie folgt:

[H]auptsächlich (...) Migrantinnen und Migranten sowie Jugendliche mit schulischen Defiziten oder Schwierigkeiten (...) [sowie] [s]chulentlassene Jugendliche, die noch nicht in der Lage sind, eine Berufsausbildung zu absolvieren (z. B. wegen Bildungsdefiziten, Schulschwierigkeiten, mangelnder geistiger und seelischer Reife, ungenügender Kenntnis der regionalen Landessprache usw.). ${ }^{4}$

Dagegen zeigt das «Lehrstellenbarometer» eine lange «Warteschlange» von Jugendlichen, welche den Eintritt in eine zertifizierende Ausbildung der Sekundarstufe II auf das Folgejahr verschieben müssen (BBT 2006; SBFI 2014). Die Warteschlange ist seit 2007 zwar kürzer geworden, entspricht vom Umfang her aber nach wie vor gut einem Sechstel eines Jahrgangs von Schulentlassenen. ${ }^{5}$ Die Pufferfunktion der Brückenangebote dürfte somit bedeutender sein, als dies der bildungspolitische Diskurs nahe legt.

\section{2 Übertritt in Brückenangebote}

Ausgehend von der Kompensations- und Berufswahlfunktion wäre zu erwarten, dass der Direkteinstieg in eine zertifizierende Sek.-II-Ausbildung vor allem Jugendlichen mit schulischen Defiziten und/oder beruflichen Orientierungsschwierigkeiten verwehrt bleibt. Diese würden sich dann auch entsprechend gehäuft in Brückenangebote

4 Diese Definition ist in der 2006 aktualisierten Version des Dokuments enthalten, die auf der SBFI-Webseite aufgeschaltet ist (www.sbfi.admin.ch/dokumentation/00335/00400/, abgerufen am 8.12.2014).

5 Anzahl Schulentlassene pro Jahr gemäss dem Tabellenanhang zu Babel et al. (2013). 
verwiesen sehen. Die bisher fundierteste Studie zur Selektion in Brückenangebote in der Schweiz (Bayard 2011) stellt demgegenüber andere Ursachen in den Vordergrund. Nach den subjektiven Gründen für den Eintritt in ein Brückenangebot (Bayard 2011, 141 ff.) gefragt, nennen die Betroffenen vor allem das Streben nach Unterstützung und verbesserten Chancen bei der Lehrstellensuche. Kompetenzdefizite sowie Orientierungsschwierigkeiten bleiben hingegen in der Wahrnehmung der Jugendlichen sekundär. Obwohl positive Aspekte nachträglich meist stärker gewichtet werden (Bayard 2011, 145; Henkel und Mather 2007; Beicht 2009, 8), wird das Durchlaufen eines Brückenangebots von rund einem Sechstel der Betroffenen explizit als sinnlose Warteschlaufe eingestuft (Bayard 2011, 147), was die Bedeutung der systemischen Pufferfunktion unterstreicht. Multivariate Analysen der Selektionsprozesse an der ersten Schwelle weisen darauf hin, dass schulische Defizite, geringe kognitive Kompetenzen sowie berufliche Orientierungsschwierigkeiten wenig zur Erklärung des direkten Übertritts in die berufliche Grundbildung beitragen (Bayard 2011, $132 \mathrm{ff}$.). Als relevant erweisen sich vielmehr der Migrationshintergrund und das Geschlecht, der auf Sekundarstufe I besuchte Schultyp sowie nicht-kognitive Kompetenzen und Kompetenzzuschreibungen (z. B. bezüglich Selbstwert). Mit Blick auf die erstgenannten Aspekte stimmen die Ergebnisse von Bayard mit früheren Befunden überein (Haeberlin et al. 2004; Imdorf 2007; Hupka et al. 2010; Hupka-Brunner et al. 2011).

Insgesamt spricht die Forschung bezüglich Geschlecht und Migrationshintergrund dafür, dass junge Frauen und darunter besonders jene mit Migrationshintergrund beim Übertritt in eine berufliche Grundbildung auf höhere Hürden stossen. Zudem finden sich auch Indizien für eine ausgeprägte Selektivität nach der sozialen Herkunft, die sich zum Nachteil von Jugendlichen aus bildungsfernen, mit wenig Sozialkapital ausgestatteten Familien auswirkt (insb. Haeberlin et al. 2004; Hupka et al. 2010, 16f.).

Als wesentliche Grundlage der betrieblichen Lehrlingsselektion und damit der individuellen Chancen auf einen Direkteinstieg in die Sekundarstufe II erweist sich in der Forschung der absolvierte Schultyp der Sekundarstufe I (dazu auch Herzog et al. 2004, 18). Die Zuweisung zu den in vielen Kantonen wenig durchlässigen unterschiedlichen Leistungszügen der Sekundarstufe I erfolgt dabei bereits nach dem fünften oder sechsten Schuljahr. Die Mehrzahl der Kantone kennt dabei getrennte Leistungszüge für Schülerinnen und Schüler, welche nach offizieller Sprachregelung schulisch lediglich "Grundansprüche» erfüllen, sowie für Jugendliche, die «erweiterten Ansprüchen» genügen. Viele Kantone führen überdies einen separaten Progymnasialzug. Es ist vielfach nachgewiesen, dass ein solch frühes "Tracking" den Einfluss der sozialen Herkunft auf den späteren Bildungserfolg deutlich verstärkt (z. B. Bauer und Riphahn 2006; Bauer und Riphahn 2007; Pfeffer 2008; Falter und Wendelspiess Chávez Juárez 2011; Kerr et al. 2013). 
Die frühe Zuweisung zu den verschiedenen Schultypen der Sekundarstufe I nimmt in der Schweiz so schon in hohem Masse vorweg, welche Ausbildungen den Jugendlichen nach der obligatorischen Schule offen stehen. Insbesondere Jugendliche, die einen Schultyp der Sekundarstufe I mit "Grundansprüchen» absolviert haben, finden auf Sekundarstufe II auch unter Kontrolle der schulischen Leistungen und Grundkompetenzen nur sehr beschränkt Zugang zu anspruchsvolleren Berufen (BFS/ TREE 2003, 43 f.; Hupka et al. 2010). Die strukturell verfestigte Warteschlange an der ersten Schwelle (vgl. Fussnote 5) hat überdies dazu geführt, dass Schulentlassene der Schultypen mit "Grundansprüchen» auch bei der Vergabe von Lehrstellen in weniger attraktiven Lehrberufen vermehrt durch Bewerberinnen und Bewerber mit «erweitertem» Leistungsausweis konkurrenziert werden (BBT 2006, 83 f.). Dies verschärft die Platzierungsprobleme von Schulentlassenen, die nur "Grundansprüche» erfüllen - und damit die soziale Selektivität.

Meyer (2009) spricht in diesem Zusammenhang von Bildungsrationierung: Demnach enthalten die Sek.-I-Schulzüge mit eingeschränkten Curricula («Grundansprüche») den Jugendlichen systematisch Lerngelegenheiten vor, was sich nachteilig auf die nachobligatorische Ausbildungslaufbahn auswirkt. Diese Nachteile dürften durch statistische Diskriminierungen seitens der Lehrbetriebe verstärkt werden, die bei der Vergabe von Lehrstellen systematisch Jugendliche aus Schulzügen mit erweiterten Anforderungen bevorzugen (siehe z. B. Haeberlin et al. 2004; Helland und Støren 2006). Entsprechend finden Hupka-Brunner et al. (2011) in Kantonen mit einem hohen Anteil des Sek.-I-Schultyps mit «Grundansprüchen» auch deutlich mehr Jugendliche, die sich nach dem Abschluss der obligatorischen Schulzeit auf ein Brückenangebot verwiesen sehen. Wo die Bildungsrationierung im definierten Sinne viele Jugendliche trifft, zieht dies einen entsprechenden Bedarf an kompensatorischen Massnahmen in Form verschiedener Übergangslösungen nach sich.

Die soweit berichteten Forschungsergebnisse zum (Direkt-)Einstieg in die berufliche Grundbildung stimmen gut mit den Beobachtungen von Meyer (2003) überein, wonach die Übervertretung von jungen Frauen, von Jugendlichen aus bildungsfernen, sozial weniger gut gestellten Familien und/oder solchen mit Migrationshintergrund, wie sie für die Sek.-I-Schultypen mit «Grundansprüchen» typisch ist, sich auch innerhalb der Brückenangebote fortschreibt.

\subsection{Wirkung von Brückenangeboten im weiteren Ausbildungsverlauf}

Bei der Untersuchung der Wirkungen von Brückenangeboten im nachobligatorischen Bildungsverlauf fokussieren wir auf die bildungspolitisch relevantesten Aspekte, nämlich auf den Eintritt in eine zertifizierende Sek.-II-Ausbildung und auf deren erfolgreichen Abschluss. Aus theoretischer Sicht ist dabei aus verschiedenen Gründen anzunehmen, dass sich die Chancen, in eine Ausbildung der Sekundarstufe II einzutreten - und damit indirekt letztlich auch die Chancen, sie erfolgreich 
abzuschliessen - verschlechtern, wenn der Übertritt nicht unmittelbar nach dem Verlassen der obligatorischen Schule gelingt:

, Zum einen ist die Annahme einer statistischen Diskriminierung (vgl. Helland und Støren 2006; Müller und Wolter 2014) jener Jugendlichen plausibel, die nicht direkt in eine zertifizierende Sek.-II-Ausbildung einsteigen. Ein ausbleibender Direkteinstieg kann dabei von potenziellen Lehrbetrieben aus zwei Gründen als negatives Signal interpretiert werden. Erstens kann er signalisieren, dass andere Betriebe triftige Gründe hatten, die Jugendlichen nicht zu berücksichtigen. Zweitens kann ein ausgebliebener Direkteinstieg auch als Signal für ungenügende Qualifikationen, fehlende Motivation oder andere ungünstige Charakteristika gedeutet werden. Beides beeinträchtigt die Chancen der betroffenen Jugendlichen bei der weiteren Lehrstellensuche. Zum anderen ist zu vermuten, dass sich eine erfolglose Lehrstellensuche negativ auf die psychischen Ressourcen der betroffenen Jugendlichen wie Selbstwert, Selbstwirksamkeit oder ausbildungsrelevante Selbstkonzepte auswirkt (Neuenschwander 2006; Herzog et al. 2006). Solche Ressourcen sind erforderlich, wenn es darum geht, sich neu zu orientieren oder die Suche nach einer Lehrstelle neu aufzunehmen.

Unter beiden Aspekten gleicht der unfreiwillig verzögerte Übertritt an der ersten Schwelle einer Arbeitslosigkeitserfahrung beim Übergang in den Arbeitsmarkt (zweite Schwelle). Die negativen Wirkungen solcher «Einstiegsarbeitslosigkeit» für den weiteren Erwerbsverlauf der Betroffenen sind gut belegt (zum Überblick: Scarpetta et al. 2010, 16; Vandenberghe 2010). Diese sogenannten "Scarring"-Effekte scheinen dabei im postulierten Sinne teilweise auf negativen psychischen Folgen von früher Arbeitslosigkeit zu beruhen (Furnham 1985; Clark et al. 2001; Bell und Blanchflower 2010).

Anzufügen ist, dass unsere Argumente auf jene Jugendlichen fokussieren, die trotz Bemühungen keinen Ausbildungsplatz finden. Daneben gibt es auch eine kleinere Gruppe von Schulentlassenen, die freiwillig in Übergangslösungen eintreten. Dabei dürfte es sich teils um Jugendliche mit fehlender «Berufswahlreife», teils auch um relativ privilegierte Jugendliche handeln, die sich z. B. besser auf eine spezifische Wunschausbildung vorbereiten wollen, in der sie auf Anhieb keinen Ausbildungsplatz erhalten haben (siehe Bayard 2011). Bei diesen Jugendlichen sind nicht unbedingt negative Wirkungen auf den weiteren Ausbildungsverlauf zu erwarten. Allerdings dürfte es sich um eine Minderheit handeln, sodass im Aggregat negative Wirkungen zu erwarten sind.

Brückenangebote können den postulierten negativen Folgen eines verzögerten Einstiegs im Prinzip auf unterschiedliche Weise entgegenwirken, wobei es eine empirische Frage ist, inwieweit dies auch gelingt. Gemäss den erwähnten Befunden erweisen sich viele Brückenangebote aus der Sicht der Jugendlichen primär wegen 
der direkten Unterstützung bei der Suche nach einem Ausbildungsplatz als wertvoll. Sie können aber auch zur Stärkung von psychischen Ressourcen beitragen, die bei der Suche nach einem Ausbildungsplatz wichtig sind, bei der Behebung von allfälligen schulischen Lücken und Kompetenzdefiziten helfen oder Unterstützung bei der Berufswahl bieten.

Obschon somit vielfältige Möglichkeiten zur Unterstützung betroffener Jugendlicher bestehen, ist nicht unbedingt zu erwarten, dass Brückenangebote die postulierten negativen Wirkungen des verzögerten Einstiegs voll kompensieren können. Zum einen ist dies bezüglich der psychischen Wirkung eher fraglich. Zum anderen können die Anbieter von Brückenangeboten auch kaum etwas an einer allfälligen negativen Signalwirkung im Lehrstellenmarkt ändern.

Einiges spricht somit für die Annahme, dass die Bildungschancen durch einen verzögerten Übergang an der ersten Schwelle nachhaltig beeinträchtigt werden. Bislang fehlt es allerdings an kausalanalytischen Studien, welche die systematische Selektion beim Eintritt in Brückenangebote angemessen kontrollieren (vgl. Gangl 2010). Der Forschungsstand beschränkt sich derzeit hauptsächlich auf deskriptive Ergebnisse zu gruppenspezifischen Übertrittsquoten (Gertsch et al. 1999; Meyer 2003; Niederberger 2003; Tondorf und Schuppli 2012). Am elaboriertesten sind die multivariaten Analysen von Bayard (2011), wonach jungen Frauen, Jugendlichen aus Schultypen mit Grundanforderungen, aus Familien mit einem tiefen sozioökonomischen Status sowie solchen mit verspäteter Einschulung und/oder Repetitionen der Übertritt von einem Brückenangebot in eine zertifizierende Ausbildung ceteris paribus seltener gelingt. Dem Übertritt förderlich sind laut Bayard dagegen nichtkognitive Kompetenzen wie Selbstwert oder Ressourcenmanagement.

Verschiedene Analysen liefern zudem Indizien, dass ein verzögerter Einstieg sich auch unter relativ umfassender Kontrolle relevanter Individual- und Kontextmerkmale negativ auf die Chance auswirkt, später noch Zugang zu einer Sek.-II-Ausbildung zu finden (Hupka-Brunner et al. 2011; Sacchi et al. 2011). In Kantonen mit besonders ausgeprägter Bildungsrationierung im Sinne von Meyer (2009) tragen Schulentlassene zudem ein höheres Risiko, auch im zweiten Jahr nach dem Austritt aus der obligatorischen Schule ohne Zugang zu einer zertifizierenden Sek.-II-Ausbildung zu verbleiben (Hupka-Brunner et al. 2011).

Bezüglich der Wirkungen auf den Abschluss einer Sek.-II.-Ausbildung vermuten wir, dass diese hauptsächlich auf der oben skizzierten statistischen Diskriminierung und einem psychischen "Scarring"-Effekt beruhen, welche den Einstieg in eine Sek.-II-Ausbildung erschweren. Allerdings sind auch länger anhaltende psychische Wirkungen nicht auszuschliessen, mit denen sich das Risiko erhöht, dass eine verzögert begonnene Ausbildung vorzeitig wieder abgebrochen wird. Empirische Befunde zu Wirkungen auf die Abschlusschancen fehlen dabei völlig. Laut deskriptiven Ergebnissen von Meyer und Bertschy (2011) bleiben Schulentlassene, welche 
ein Brückenangebot absolvieren, rund doppelt so häufig ohne Sek.-II-Abschluss wie Direkteinsteigerinnen und -einsteiger in zertifizierende Ausbildungen.

Generell erlauben die spärlichen Forschungsergebnisse bislang keine Rückschlüsse auf kausale Wirkungen von Brückenangeboten auf den späteren Bildungsverlauf. Das liegt teils daran, dass ausschliesslich Brückenangebots-Absolventen einbezogen werden, teils an einer fehlenden Kontrolle von potenziell konfundierenden Drittfaktoren, welche sowohl die Art des Übergangs als auch den nachobligatorischen Bildungserfolg beeinflussen.

\section{Hypothesen}

\subsection{Hypothesen zu den Selektionsprozessen beim Übergang in Brückenangebote}

Unsere erste Fragestellung zielt auf die individuellen, familiären und kontextuellen Faktoren, welche über den direkten Einstieg in eine nachobligatorische Ausbildung, das Ausweichen auf ein Brückenangebot oder das gänzliche Ausscheiden aus dem Bildungssystem entscheiden. Ausgehend von Abschnitt 2.2 formulieren wir dazu folgende Hypothesen:

, Die postulierte Kompensationsfunktion lässt annehmen, dass vor allem Schulentlassene in Brückenangebote eintreten, die im Vergleich zu Direkteinsteigerinnen und -einsteigern schwächere schulische Leistungen aufweisen. Dasselbe gilt sinngemäss für nicht-kognitive Kompetenzen (wie z. B. Selbstkonzepte) sowie für Sekundärtugenden (wie z. B. Pünktlichkeit), die bei der Suche nach einer Lehrstelle wichtig sind.

, Die Orientierungs-bzw. Berufswahlfunktion legt dagegen nahe, dass vor allem Jugendliche in Brückenangebote eintreten, die am Ende der neunten Klasse noch keine Klarheit darüber gewonnen haben, welche Ausbildungs- und Berufsziele sie anstreben möchten.

, Mit Blick auf die systemische Puffer-Funktion ist zu erwarten, dass sich ein ungenügendes Lehrstellenangebot selektiv zu Ungunsten von spezifischen Gruppen von Schulentlassenen auswirkt. Dazu zählen in erster Linie junge Frauen sowie Jugendliche, die einen Sek.-I-Schultyp mit «Grundanforderungen» besucht haben, die aus bildungsfernen Familien mit wenig Sozialkapital kommen oder die einen Migrationshintergrund aufweisen.

Angesichts der hohen institutionellen Heterogenität bezüglich der kantonalen Ausgestaltung der Sekundarstufe I, des Systems von Brückenangeboten sowie der Verfassung und Struktur des Lehrstellenmarkts rechnen wir mit bedeutenden bildungsräumlichen Disparitäten. In Kantonen mit ausgeprägter Bildungsrationierung und einem entsprechend hohen Anteil an Lernenden in Sek.-I-Schultypen mit "Grundanforderungen» erwarten wir einen über- 
proportionalen Anteil an Jugendlichen, die Übergangslösungen durchlaufen (Hupka-Brunner et al. 2011, 182).

- Schliesslich ist auch die Annahme plausibel, dass die soziale Unterstützung durch Elternhaus und Schule einen nahtlosen Übergang an der Schwelle zur Sekundarstufe II begünstigt (vgl. Neuenschwander 2008).

\subsection{Hypothesen zur Wirkung von Brückenangeboten auf den weiteren Bildungserwerb}

Unsere zweite Fragestellung zielt darauf, wie sich ein verzögerter Einstieg bzw. das Durchlaufen von Brückenangeboten bei gegebenen individuellen, familiären und schulischen Voraussetzungen auf die Chancen von Jugendlichen auswirkt, in eine zertifizierende nachobligatorische Ausbildung einzutreten und diese auch erfolgreich abzuschliessen.

Im Einzelnen formulieren wir diesbezüglich folgende Hypothesen:

- Wenn es Schulentlassenen nicht gelingt, direkt in eine zertifizierende nachobligatorische Ausbildung einzusteigen, reduziert sich ihre Chance, später noch in eine solche einzutreten und diese erfolgreich abzuschliessen.

- Wenn der Direkteinstieg nicht gelingt, so vermögen Brückenangebote die dadurch bedingte Reduktion der Einstiegschancen nicht vollumfänglich zu kompensieren. Die Chancen der betroffenen Jugendlichen bleiben nachhaltig beeinträchtigt.

- Institutionell gut verankerte Brückenangebote wie insbesondere das zehnte Schuljahr sind besser als andere Übergangslösungen in der Lage, negativen Folgen des verzögerten Einstiegs entgegenzuwirken.

\section{Empirische Analyse}

\subsection{Datenbasis}

Mit der Datenbasis von TREE (Transitionen von der Erstausbildung ins Erwerbsleben) ist es in der Schweiz erstmals möglich, die zahlreichen als übergangsrelevant erachteten Faktoren in einem umfassenden multivariaten Selektionsmodell zu berücksichtigen. Dank dem langen Beobachtungszeitraum kann zudem untersucht werden, wie sich das Durchlaufen von Brückenangeboten auf die Chance auswirkt, einen Abschluss der Sekundarstufe II zu erwerben.

TREE ist eine Follow-up-Befragung der PISA 2000-Erhebung in der Schweiz. Die Stichprobe umfasst gut 6000 Jugendliche, welche an der PISA 2000-Befragung teilnahmen und die obligatorische Schule im selben Jahr verlassen haben. Die Stichprobe wurde bisher insgesamt acht Mal nachbefragt, von 2001 bis 2007 
im Jahresrhythmus. Für die vorliegende Analyse berücksichtigen wir die bis 2007 erhobenen Daten (Stalder et al. 2011).

Bei der Analyse der Übertrittsprozesse schliessen wir diejenigen Jugendlichen aus, die ein Jahr nach Erfüllung der Schulpflicht ein Gymnasium oder eine Lehrkräfteausbildung besucht haben. ${ }^{6}$ Die Stichprobe umfasst so noch 3607 Jugendliche. Dabei unterscheiden wir zwischen Jugendlichen, die im ersten Jahr nach dem Austritt aus der obligatorischen Schule in einer zertifizierenden Sek.-II-Ausbildung (berufliche Grundbildung mit EFZ, Handels-, Diplom- oder Fachmittelschule, $\mathrm{n}=2663)$ sind, solchen, die ein Brückenangebot absolvieren $(\mathrm{n}=796)$, sowie solchen $(\mathrm{n}=146)$, die nach Schulaustritt keinerlei Ausbildungsaktivitäten ausüben. Innerhalb der Brückenangebote unterscheiden wir zusätzlich zwischen dem 10. Schuljahr als institutionell gut verankerter und im Sample quantitativ gut besetzter Kategorie $(\mathrm{n}=592)$ und einer heterogenen Restkategorie, die etwa Sprachaufenthalte, Praktika oder Au-Pair-Stellen umfasst $(\mathrm{n}=204)$.

Bei der Analyse der Wirkungen der Art des Übertritts stützen wir uns auf dasselbe Sample, wobei sich der Sampleumfang wegen der Panelattrition allerdings reduziert. Als abhängige Variablen ziehen wir einerseits den Abschluss einer zertifzierenden Sek.-II-Ausbildung innert sechs Jahren nach Schulaustritt heran (sechste TREE-Welle, verbleibendes $n=2403$ ). Die TREE-Kohorte war am Ende dieses Beobachtungszeitraums im Mittel rund 21 Jahre alt und der Bildungserwerb auf Sek.-II-Stufe weitestgehend zum Abschluss gekommen. Andererseits untersuchen wir auch die Wirkungen auf den Eintritt in eine anerkannte Sek.-II-Ausbildung bis zum zweiten Jahr nach dem Austritt aus der obligatorischen Schule (zweite TREEWelle), d. h. im Alter von durchschnittlich etwa 17 Jahren. Dies ist nicht nur wegen des wesentlich grösseren Analysesamples $(n=3154)$ sinnvoll, sondern weil so erkennbar wird, wie sich die anfängliche Wirkung eines verzögerten Einstiegs im weiteren Bildungsverlauf verändert. Theoretisch sind sowohl verstärkende als auch kompensatorisch wirkende Prozesse denkbar.

\subsection{Methoden}

Um den Einfluss der oben aufgeführten individuellen, familiären, schulischen und kontextuellen Merkmale auf den Übergang von der obligatorischen Schule in die beiden betrachteten Typen von Zwischenlösungen (10. Schuljahr vs. andere Übergangslösungen) zu ermitteln, schätzen wir ein multinomiales Logit-Modell (Hosmer und Lemeshow 2000 [1989]). Die Jugendlichen mit einem direkten, unverzögerten Übergang von der neunten Klasse in eine zertifizierende Sek.-II-Ausbildung bilden dabei die Referenzgruppe, mit der jene Jugendlichen verglichen werden, die in einen der beiden betrachteten Typen von Brückenangeboten (10. Schuljahr, andere Zwischenlösung) übertreten. Als zusätzliche Vergleichsgruppe berücksichtigen wir

$6 \quad$ Ferner schliessen wir auch Jugendliche aus, die eine Anlehre $(n=13)$, eine Vorlehre $(n=55)$ oder Vorkurse/Passerellen für zertifizierende Sek.-II-Ausbildungen $(\mathrm{n}=35)$ absolvieren. 
Jugendliche, die nach der obligatorischen Schule vorübergehend oder dauerhaft aus dem Bildungssystem ausscheiden. Der Vergleich dieser "Drop-Outs" mit Jugendlichen, die ein Brückenangebot durchlaufen, ermöglicht es, bei der anschliessenden Wirkungsanalyse mittels Propensity Score Matching zwischen den Folgen des verzögerten Einstiegs einerseits und den Wirkungen von Brückenangeboten andererseits zu unterscheiden. Da die Kategorie der "Drop-Outs" im Analysesample relativ schwach besetzt ist $(\mathrm{n}=146)$, ist die Schätzgenauigkeit hier leicht reduziert (vgl. Standardfehler in Tabelle 2).

Als Prädiktoren können wir auf aussergewöhnlich umfassende Messungen von Individual-, Familien- und Kontextmerkmalen zurückgreifen, von denen gemäss Abschnitt 2.2 anzunehmen ist, dass sie den interessierenden Übertrittsprozess beeinflussen. Diese sind allesamt der am Ende der obligatorischen Schulzeit durchgeführten PISA-Erhebung entnommen. Die Prädiktorenauswahl umfasst Indikatoren für die schulischen Leistungen (PISA-Kompetenzmessungen in Lesen und Mathematik, Noten), für nicht-kognitive Kompetenzen und relevante Motivationen, für berufliche Orientierungsschwierigkeiten, zum räumlich-institutionellen Kontext sowie Angaben zur sozialen Herkunft, zum Migrationshintergrund und zum Geschlecht. Eine vollständige Liste der verwendeten Prädiktoren inklusive Angaben zur Operationalisierung findet sich in der Tabelle A1 im Anhang, eine übersichtsartige Darstellung in der Abbildung 1.

Das multinomiale Logit-Modell schätzen wir mit einer Stichprobengewichtung, die das disproportionale Design der TREE-Ausgangsstichprobe sowie den massgeblichen Stichprobenschwund bis zur ersten TREE-Welle korrigiert (Sacchi 2011).7 Neben dem Unit-Nonresponse ist auch der bei einigen Indikatoren beträchtliche Item-Nonresponse ein Problem. Bei einem listenweisen Ausschluss von Fällen mit fehlenden Angaben würde sich das Analyse-Sample markant verkleinern. ${ }^{8}$ Um den damit verbundenen Verlust an Informationen und Teststärke zu vermeiden, ist bei den dargestellten Ergebnissen ein multiples Imputationsverfahren eingesetzt worden (Rubin 1987; Acock 2005). ${ }^{9}$ Wir schätzen daher auf der Basis von 10 mittels Imputation vervollständigter Replikationssamples zunächst ein vollständiges Modell mit sämtlichen Prädiktoren. Anschliessend führen wir eine vorsichtige zweistufige Rückwärtsbereinigung durch - zunächst auf der Ebene des Modells, dann auf der Ebene der einzelnen Schätzgleichungen. ${ }^{10}$ Das so gewonnene Modell umfasst nur

$7 \quad$ Die Ergebnisse ohne Nonresponse-Korrektur unterscheiden sich nur marginal (Sacchi 2011, 44).

8 Der Anteil der imputierten Werte ist in der Anhangtabelle A1 ausgewiesen; Fälle mit mehr als zwei fehlenden Angaben sind aber selten (weniger als 3 Prozent).

9 Gestützt auf Rubin $(1996,480)$ entscheiden wir uns für 10 Replikationssamples (generiert mit ICE, vgl. Royston 2004; Royston 2007) und kombinieren die 10 Modellschätzungen dann anhand der "Rubin-Rules".

10 Zuerst schliessen wir jene Variablen aus, für die die drei zugehörigen Effektkoeffizienten (B) weder gemeinsam ( $\mathrm{p}$-Wert $>0.15$ ) noch einzelnen getestet $(\mathrm{p}-$ Wert $>0.10)$ signifikant sind. Anschliessend bereinigen wir die drei Schätzgleichungen mittels Restringierung um nicht-signifikante Effekte ( $\mathrm{p}$-Wert > 0.15; Modelle ohne Rückwärtsbereinigung liefern wir gerne auf Anfrage). 
Abbildung 1 Postulierte Selektions- und Wirkungsprozesse und verwendete Indikatoren

Individuelle Voraussetzungen im 9. Schuljahr

\begin{tabular}{l}
\hline Indikatoren für individuelle Defizite \\
Schulleistungen und kognitive Kompetenzen \\
- Noten in Lesen und Mathematik \\
- Kompetenz in Lesen und Mathematik (PISA Scores) \\
Nicht-kognitive Kompetenzen \\
- Persistenz \\
- Selbstwirksamkeit \\
- Kontrollüberzeugungen \\
- Verbales und mathematisches Selbstkonzept \\
Motivationale Aspekte \\
- Instrumentelle Motivation \\
- Erwarteter Beruf mit 30 (ISEI) \\
- Häufigkeit von Absenzen \\
\hline
\end{tabular}

Indikatoren für Berufswahlschwierigkeiten

Ausbildungspläne und Laufbahnorientierung

- Ausbildungspläne nach obl. Schule (weiss nicht)

- Beruf mit 30 (weiss nicht)

Indikatoren der institutionellen Heterogenität

Räumlich-institutioneller Kontext

- Kantonaler Anteil des Sek I-Schultyps mit

«Grundansprüchen»

- Stadt/Land (Urbanisierungsgrad)

- Sprachregion

Indikatoren der sozialen Selektivität

- Geschlecht

- Sek. I-Schultyp

Soziale Herkunft / Migrationshintergrund

- Bildung der Eltern

- Kulturelle und finanzielle Situation

- Herkunftsland Vater

Persönliches Umfeld

- Unterstützung Eltern

- Unterstützung durch Lehrkräfte

- Elterliches Interesse (schulisch und sozial)
Nachobligatorischer Ausbildungsverlauf

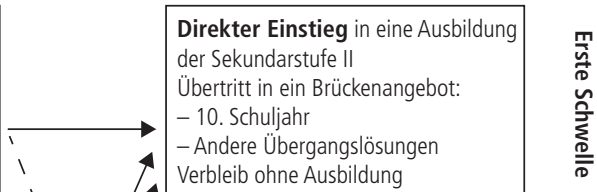

Legende:

Analyse mittels multinomialer Regression

Analyse mittels Propensity Scores Matching (PSM)

Potenziell konfundierende Einflüsse (mit PSM kontrolliert)

noch Prädiktoren, bei denen zumindest schwache Indizien vorliegen, dass sie für die betrachteten Übergänge eine Rolle spielen. Die Bereinigung des Modells und der einzelnen Schätzgleichungen um eindeutig nicht signifikante Effekte ist im Hinblick auf die anschliessende Wirkungsanalyse sinnvoll, da sie eine präzisere Schätzung der Propensity Scores und damit letztlich der interessierenden Treatment-Effekte ermöglicht. Eine Reduktion der Modellkomplexität ist zudem auch mit Blick auf die relativ schwach besetzte Kategorie der "Drop-Outs" vorteilhaft. 
Bei der Berechnung der Standardfehler und Signifikanzen stellen wir die komplexe Struktur des TREE-Samples in Rechnung. ${ }^{11}$ Neben den Effektkoeffizienten, den zugehörigen Standardfehlern und Signifikanztests weisen wir im empirischen Teil zu jeder Variable auch einen Test der Hypothese aus, dass die zugehörigen Effektkoeffizienten in den drei Schätzgleichungen allesamt nicht von Null verschieden sind. Bei der Ergebnisinterpretation stützen wir uns zudem auf zusätzliche, nicht dargestellte Tests ab, mit denen wir einerseits für jeden Prädiktor prüfen, ob alle drei zugehörigen Effektkoeffizienten gleich gross sind, und andererseits, ob sich dessen Effekte für die beiden Typen von Brückenangeboten unterscheiden.

Die Wirkungen des verzögerten Einstiegs sowie des Durchlaufens der beiden betrachteten Typen von Brückenangeboten schätzen wir mittels Propensity Score Matching (vgl. z. B. Winship und Morgan 1999; Gangl und DiPrete 2004). Dies ermöglicht eine empirische Abschätzung von Kausalwirkungen mit nichtexperimentellen Daten. Die Methode beruht im Kern auf einem Vergleich von Personen, die einem definierten "Treatment" (z. B. einer Behandlung, einem Kurs usw.) ausgesetzt sind, mit «unbehandelten» Samplemitgliedern mit vorgängig völlig identischen Merkmalen und Ausgangsbedingungen. Dafür werden - häufig auf der Basis eines Logit-Modells - «behandelte» und «unbehandelte» Personen mit identischen «Behandlungs-Wahrscheinlichkeiten» (Propensity Scores) identifiziert. Die mittlere Differenz zwischen behandelten und unbehandelten Personen mit gleichen Propensity Scores und mithin vergleichbaren Ausgangsbedingungen, der "Average Treatment Effect on the Treated" (ATT), kann dann unter bestimmten Annahmen kausal interpretiert werden. Entscheidend ist dafür namentlich, dass bei der Schätzung der "Propensity Scores" all jene Merkmale und Bedingungen eingeschlossen werden (können), welche sowohl den Eintritt ins interessierende "Treatment" als auch die interessierende Wirkungsdimension - hier den späteren Eintritt und Abschluss einer Sek.-II-Ausbildung - beeinflussen ("Conditional Independence Assumption" [CIA]). ${ }^{12}$ Diesbezüglich spricht der Forschungsstand dafür, dass etliche der Merkmale und Bedingungen, die gemäss Abschnitt 2.2 für den Übertritt an der ersten Schwelle relevant sind, auch den Verbleib ohne nachobligatorischen Ausbildungsabschluss beeinflussen (vgl. Solga 2005; BIBB 2009; Ulrich 2013). Da die TREE-Daten eine aussergewöhnlich umfassende Kontrolle all dieser Merkmale erlauben, dürfte die CIA-Annahme hier in sehr guter Näherung erfüllt sein. Das Modell zur Schätzung der Propensity Scores darf dabei durchaus noch weitere Variablen beinhalten, welche ausschliesslich die Selektion ins "Treatment" beeinflussen (Stuart 2010, 5). Die benötigten Propensity Scores können wir daher unmittelbar der multinomialen

11 Die Berechnung der Standardfehler (STATA-Prozedur «Svy: Mlogit») berücksichtigt die Stichprobengewichtung und das komplexe Sampling (zweistufige, mehrfach stratifizierte Auswahl, Endlichkeitskorrektur, vgl. Sacchi 2011).

12 Grundlegend ist ferner auch die "Stable Unit Treatment Value Assumption" (vgl. Gangl und DiPrete 2004, 402). 
Modellierung der Übergangsprozesse entnehmen. ${ }^{13}$ Für die darauf beruhende Schätzung der Treatment-Effekte verwenden wir ein "10-Nearest-Neighbor"-Verfahren, bei dem jedem Jugendlichen in einer der drei Treatment-Gruppen (10. Schuljahr, anderes Brückenangebot, ohne Anschlusslösung) jeweils (maximal) zehn direkt in eine zertifizierende Ausbildung eingestiegene Jugendliche mit vergleichbaren Merkmalen am Ende der obligatorischen Schule zugeordnet werden. ${ }^{14}$ Um die Qualität des Matching zu prüfen, haben wir verschiedene Balancing-Tests durchgeführt; diese sprechen für durchwegs sehr geringe Unterschiede zwischen den "Treated” und den gematchten "Controls". ${ }^{15}$

Da wir an einem Populationsschätzwert des mittleren Treatment-Effekts (ATT) interessiert sind, ziehen wir für die Schätzung die passenden Stichprobengewichtungen heran (vgl. DuGoff et al. 2014, 289). Wir weisen dabei Ergebnisse auf der Basis von zwei unterschiedlichen Stichprobengewichten aus: Einer reinen Designgewichtung, welche einzig die je nach Stratum sehr ungleichen Auswahlsätze der PISA-Erhebung kompensiert, sowie einer Gewichtung, die zusätzlich eine Korrektur der späteren Panelattrition beinhaltet. ${ }^{16}$ Die Standardfehler und Vertrauensintervalle der ATT bestimmen wir mit dem blockweisen Bootstrap-Verfahren von de Luna et al. (2010, Absatz 3.2). ${ }^{17}$

\subsection{Empirische Analyse}

\subsubsection{Selektionsprozesse beim Übergang in Brückenangebote}

Wir betrachten zuerst den Übergang an der ersten Schwelle und anschliessend dessen Wirkungen auf den späteren Ausbildungsverlauf. Tabelle 1 schlüsselt die TREE-Kohorte zunächst nach der Art der Anschlusslösung auf.

Rund drei Viertel der Schulentlassenen des Jahres 2000 durchlaufen demnach einen der hier näher analysierten Übergänge; vom Analysesample ausgeschlossen bleiben insbesondere Jugendliche in Gymnasien (siehe Abschnitt 4.1). Von den

13 Bei der Schätzung der Propensity Scores wird zuweilen (z. B. DuGoff et al. 2014, 289) von der Verwendung von Survey-Gewichten abgeraten, ebenso vom Ausschluss von Variablen, die nur das "Outcome" beeinflussen (Brookhart et al. 2006; Austin 2011). In unserer Auswertung unterscheiden sich ATT und Standardfehler nicht nennenswert, wenn wir die Propensity Scores ohne Gewichtung und ohne Rückwärtsbereinigung schätzen (Ergebnisse auf Anfrage).

14 Für die Schätzung ziehen wir "psmatch2» (Leuven und Sianesi 2003) heran, wobei wir einen Caliper-Parameter definieren (gemäss Gangl und DiPrete 2004, 413) und die Analyse auf den gemeinsamen Support einschränken.

15 Die Mittelwert-Differenzen der standardisierten Propensity-Scores sind vernachlässigbar $(\leq 0.001)$ und das Verhältnis ihrer Varianzen bewegt sich innerhalb des wünschbaren Bereichs (zwischen 1.05 und 1.65; vgl. Stuart 2010, 11). Bivariate Tests zeigen nur vereinzelt Abweichungen, die aufgrund der zahlreichen Tests zu erwarten sind.

16 Für den Ausbildungseintrittziehen wir das Gewicht der zweiten Befragungswelle heran («wt2_kal»). Für den Ausbildungsabschluss konstruieren wir ein massgeschneidertes Gewicht (gemäss Sacchi 2011, 44).

17 Anders als konventionelle Bootstrap-Verfahren (Abadie und Imbens 2008) ermöglicht dies bei Nearest-Neighbor-Verfahren eine konsistente Schätzung der Samplevarianz (unter Einbezug der Stichprobenstratifizierung). 
Tabelle 1 Analysierte Übergänge an der ersten Schwelle

\begin{tabular}{lrrr}
\hline Art des Übergangs & \multicolumn{2}{c}{ Gewichteter Anteil (\%) } & Sampleumfang \\
& an allen Schulentlassenen & am Analyse-Sample & $\mathrm{n}$ \\
\hline Direkteinstieg & 52.6 & 70.0 & 2663 \\
10. Schuljahr & 13.9 & 18.5 & 592 \\
Andere Übergangslösungen & 4.4 & 5.8 & 204 \\
Ohne Anschlusslösung & 4.2 & 5.6 & 146 \\
Total & 75.1 & 100.0 & 3605 \\
\hline
\end{tabular}

Anmerkungen: Gewichtung der Anteilswerte inkl. Nonresponse-Korrektur («wt1_kal»). Sampleumfang ungewichtet. Andere Überganslösungen: Sprachaufenthalte, Praktika, Au-Pair usw.

Quelle: TREE, eigene Berechnungen.

übrigen Schulentlassenen steigen rund 70 Prozent direkt in eine zertifizierende nachobligatorische Ausbildung ein, 19 Prozent in ein 10. Schuljahr und 6 Prozent in eine andere Übergangslösung, während rund 6 Prozent (zunächst) ohne Anschlusslösung verbleiben.

Das multinomiale Modell zu den verschiedenen Übergangstypen an der ersten Schwelle ist in Tabelle 2 dargestellt. Wie entsprechende Tests belegen, beeinflussen die nach der Rückwärtsbereinigung im Modell verbleibenden Prädiktoren (siehe Abschnitt 4.2) die drei Übergänge in je spezifischer Weise. ${ }^{18}$ Die drei Übertrittsprozesse unterscheiden sich also substanziell, weshalb es eine separate Modellierung angebracht ist. Bei der Interpretation der einzelnen Ergebnisse folgen wir der Gruppierung der Einflussfaktoren aus Abbildung 1.

\section{Individuelle Kompetenzdefizite}

Ein erster, in dieser Deutlichkeit überraschender Befund geht dahin, dass sich Jugendliche, die in ein Brückenangebot übertreten, ceteris paribus weder bezüglich des (schulischen) Leistungsausweises noch hinsichtlich ihrer kognitiven Kompetenzen von Direkteinsteigern unterscheiden. Die Sprach- und Mathematiknoten sowie die mathematische Kompetenz erscheinen mangels signifikanter Effekte überhaupt nicht im bereinigten Modell, während eine geringe Lesekompetenz einzig für den Verbleib ohne Anschlusslösung relevant ist. Dieses Ergebnis bestätigt sich sogar dann, wenn wir auf eine Kontrolle des Schultyps - in dem sich sowohl Leistungsunterschiede als auch die soziale Herkunft spiegelt - verzichten. ${ }^{19}$ Mit einer Ausnahme bleiben zudem

18 Wird für jede mögliche Paarung von Schätzgleichungen die Hypothese durchwegs identischer Effekte geprüft, so resultieren $\mathrm{p}$-Werte von $\leq 0.0001$ (Mittelwert Design-Adjusted Wald-Test über 10 Replikationen).

19 Ein Einfluss von Schulnoten, Lese- und Mathematikkompetenz lässt sich bis auf einen eher schwachen negativen Effekt der letztgenannten auf den Übertritt in ein 10. Schuljahr weiterhin nicht nachweisen. 


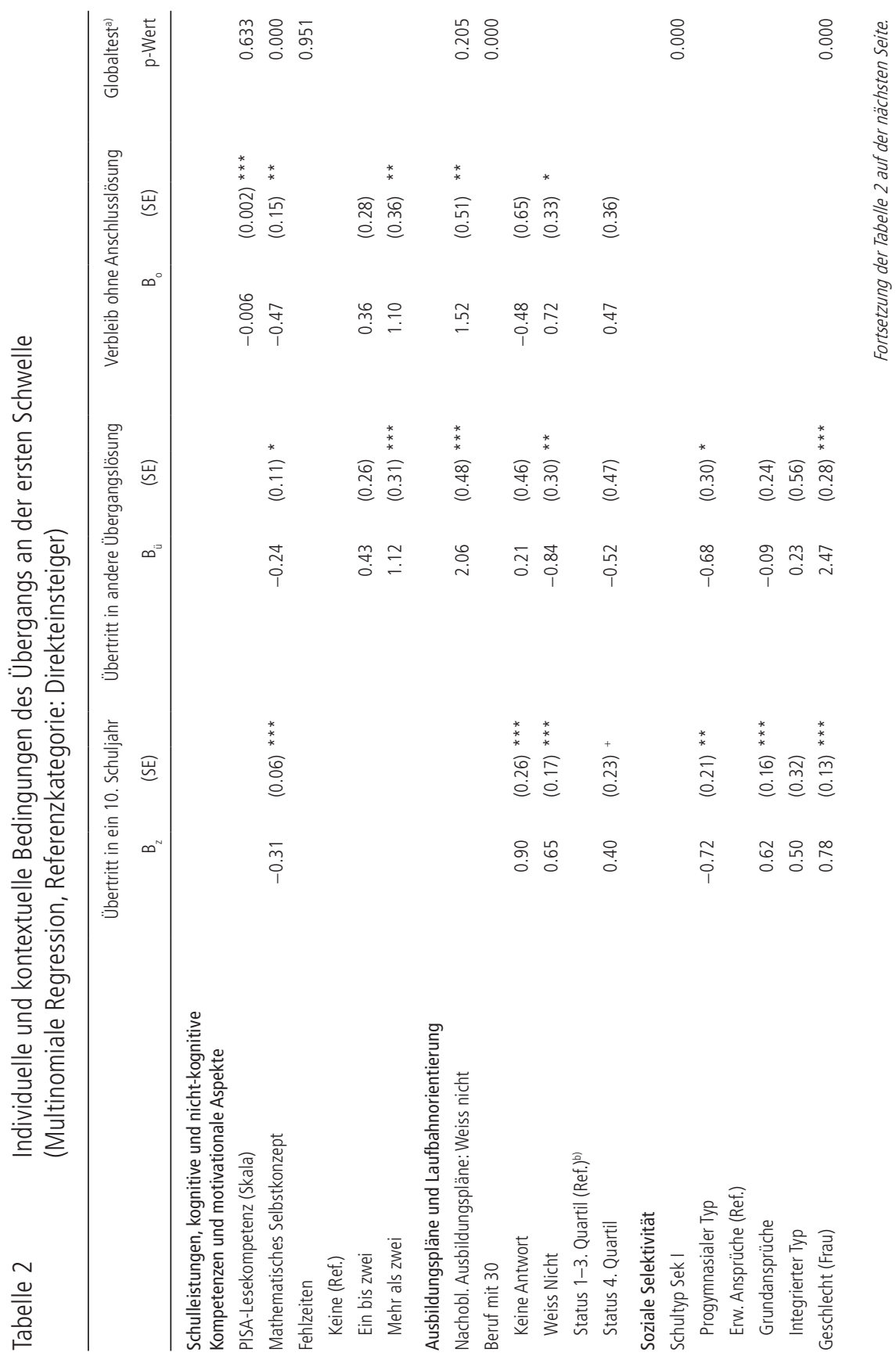




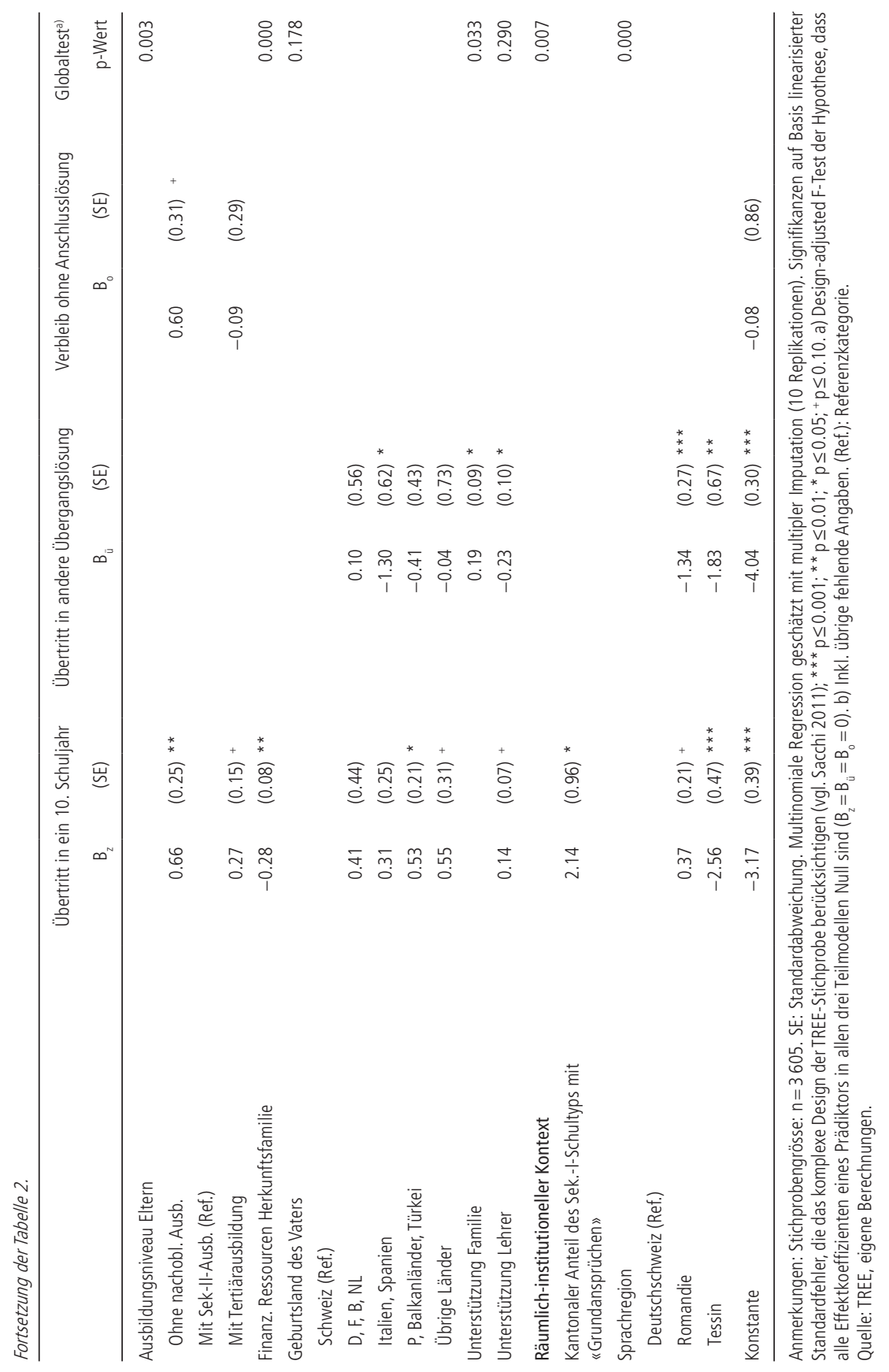


auch die nicht-kognitiven Kompetenzen, die wir kontrolliert haben (siehe Abbildung 1 und Tabelle A1 im Anhang), ohne jeden Einfluss auf den Übergang an der ersten Schwelle. Die Ausnahme bildet ein positives mathematisches Selbstkonzept, das einen direkten Einstieg in eine Sek.-II-Ausbildung begünstigt. Offenbar ist das Vertrauen in die eigenen mathematischen Fähigkeiten für einen gelingenden Direkteinstieg wichtiger als die tatsächlichen Kompetenzen, deren Effekt sich als nicht signifikant erweist. Mit Blick auf die motivationalen Indikatoren steigt bei Jugendlichen mit hohen Fehlzeiten die Wahrscheinlichkeit, dass sie in andere Übergangslösungen eintreten oder ganz ohne Anschlusslösung bleiben. ${ }^{20}$ Die übrigen einbezogenen motivationalen Aspekte spielen keine bzw. nur eine bescheidene Rolle. Insgesamt sprechen die Ergebnisse in weitgehender Übereinstimmung mit Bayard (2011, $182 \mathrm{ff}$.) dafür, dass schulische Leistungen und fehlende kognitive Grundkompetenzen im betrachteten Übergang keine bedeutende Rolle spielen.

\section{Orientierungsschwierigkeiten}

Unsere Befunde weisen darauf hin, dass bei manchen Jugendlichen unklare oder fehlende Ausbildungs- und Berufsziele den Übertritt in ein Brückenangebot motivieren. Fehlt es Jugendlichen an einem definierten Ausbildungsziel, so erhöht dies die Wahrscheinlichkeit stark, dass sie in eine Übergangslösung eintreten oder ganz ohne Anschlusslösung bleiben. Das Fehlen eines klaren Ausbildungsziels am Ende der obligatorischen Schule dürfte dabei nicht selten auch die Folge einer bis dahin vergeblichen Suche nach einem Ausbildungsplatz sein, welche eine berufliche Neuausrichtung erforderlich macht. Ein Wechselverhältnis zwischen Orientierungsschwierigkeiten und Sucherfolg ist hier plausibel. In geringerem Masse gilt das sinngemäss auch für das Fehlen eines klaren langfristigen Berufsziels («Beruf mit 30»). Dieses senkt gemäss unseren Ergebnissen die Wahrscheinlichkeit eines Übertritts in andere Übergangslösungen, begünstigt aber sehr stark den Wechsel ins zehnte Schuljahr sowie das gänzliche Ausscheiden aus dem Bildungssystem. Insgesamt stehen die Befunde somit in Einklang mit der Hypothese, wonach Jugendliche mit Orientierungsschwierigkeiten gehäuft in Brückenangebote eintreten.

\section{Soziale Selektivität}

Als sehr selektionswirksam erweist sich der auf Sekundarstufe I besuchte Schultyp, den wir aus den in Abschnitt 2.2 erläuterten Gründen in erster Linie als Indikator für die soziale Selektivität der gegliederten Sekundarstufe I interpretieren. Erwartungsgemäss tragen Jugendliche aus Schultypen mit «Grundansprüchen» unter sonst vergleichbaren Bedingungen ein fast doppelt so hohes Risiko, in ein 10. Schuljahr einzutreten, wie Jugendliche aus Schultypen mit «erweiterten» Ansprüchen $(\exp [0.62] \approx 1.9)$. Für die anderen Übergangslösungen sowie für den Verbleib ohne

20 Allerdings sind nur einzelne Koeffizienten signifikant, nicht aber der Gesamteffekt des Indikators (Tabelle 2, Spalte rechts). 
Anschlusslösung zeigt sich hingegen kein vergleichbarer Effekt. Wenig überraschend vermindert dagegen der Besuch eines Progymnasiums die Wahrscheinlichkeit eines Brückenangebots substanziell.

Neben dem Schultyp spielen auch askriptive Merkmale in den beobachteten Selektions- bzw. Allokationsprozessen eine bedeutende Rolle, insbesondere das Geschlecht und die soziale Herkunft. Im Vergleich zu den jungen Frauen haben junge Männer ceteris paribus eine markant erhöhte Chance, direkt statt verzögert in eine Sek.-II-Ausbildung einsteigen zu können. Die betreffenden Odds Ratios sind beim 10. Schuljahr um mehr als Faktor zwei $(\exp [0.78] \approx 2.2)$, bei den anderen Übergangslösungen gar um nahezu Faktor zwölf (exp[2.47] $\approx 12)$ erhöht. Darin spiegelt sich der Umstand, dass bestimmte Übergangslösungen (z. B. Au-Pair, «Welschlandjahr») fast ausschliesslich von jungen Frauen in Anspruch genommen werden. Dieser Befund ist ein deutlicher Indikator für die nach wie vor sehr ausgeprägte Geschlechtersegregation des nachobligatorischen Ausbildungssystems.

Auch die soziale Herkunft der Jugendlichen beeinflusst die Art des Übergangs in die Sekundarstufe II substanziell: Jugendliche mit mindestens einem Elternteil ohne nachobligatorischen Bildungsabschluss sowie solche aus Familien mit geringen finanziellen Ressourcen sehen ihre Chancen auf einen Direkteinstieg signifikant gemindert. Beide Effekte sind bei separater Betrachtung der drei Übergänge zwar nur teilweise signifikant, im Ganzen aber gut gesichert $(\mathrm{p} \leq 0.003$ / 0.001, siehe Tabelle 2). Die Effektunterschiede zwischen den drei Übergängen sind zudem nicht gesichert ( $p>0.35 / 0.21)$. Zusammengenommen impliziert dies, dass die Herkunft aus einer bildungsfernen Familie mit geringen finanziellen Ressourcen die Chancen auf einen Direkteinstieg generell vermindert, wohl vor allem aufgrund von Nachteilen bei der Lehrstellensuche (vgl. Hupka et al. 2010).

Weniger eindeutig sind die Ergebnisse zum Migrationshintergrund. Einerseits kann die Hypothese eines fehlenden Gesamteffekts auf die betrachteten Übergänge nicht verworfen werden $(p=0.18)$, während andererseits spezifische Effekte für einzelne Übergänge und Migrationsgruppen gut gesichert sind. Die im Vergleich zum Forschungsstand eher schwach ausgeprägten Effekte dürften mit der sehr umfassenden Kontrolle anderer Einflüsse zusammenhängen. So ist gut belegt, dass enge Zusammenhänge zwischen Migrationshintergrund, absolviertem Sek-I-Schultyp und sozioökonomischem Status bestehen (BFS/TREE 2003).

Schliesslich erweist sich auch die Unterstützung durch Schule und Elternhaus als übergangsrelevant. So deuten unsere Ergebnisse darauf hin, dass Jugendliche mit starker familiärer Unterstützung der Tendenz nach häufiger in Brückenangebote eintreten und seltener ganz ohne Anschlusslösung bleiben. Jugendliche mit viel Unterstützung durch die Lehrperson entscheiden sich eher für ein 10. Schuljahr als für eine andere Übergangslösung. 


\section{Räumlich-institutioneller Kontext}

Die Ergebnisse belegen auch die bedeutende Rolle der spezifischen Opportunitätsstrukturen, die schulentlassene Jugendliche in Funktion der institutionellen Variabilität der kantonalen Bildungssysteme vorfinden. So beeinflussen kantonale Unterschiede in der Organisation der Sekundarstufe I den Übergang in die Sekundarstufe II erheblich: Schulentlassene aus Kantonen mit einem hohen Anteil an Schülerinnen und Schülern in Sek.-I-Schultypen mit "Grundansprüchen» haben deutlich geringere Chancen, direkt in eine Sek.-II-Ausbildung einzusteigen. Bei Betrachtung der einzelnen Übergänge ist der Effekt zwar nur beim 10. Schuljahr auf konventionellem Niveau signifikant. Die Hypothese identischer Effekte für alle drei Übergänge wird allerdings dennoch nicht verworfen $(p \approx 0.81)$, und der Einfluss ist insgesamt gut gesichert $(\mathrm{p} \approx 0.007)$. Zudem ist er auch substanziell: Zwischen dem Kanton mit dem geringsten (Wallis) und dem höchsten (Bern) Anteil von Schülerinnen und Schülern aus Schultypen mit «Grundansprüchen» nimmt der erwartete Anteil an Direkteinsteigern ceteris paribus von 85 auf knapp 72 Prozent ab. ${ }^{21}$ Dies legt den Schluss nahe, dass mit dem Ausmass der Bildungsrationierung auf Sekundarstufe I im Sinne von Meyer (2009) auch der Anteil Schulentlassener steigt, denen der direkte Einstieg in eine nachobligatorische Ausbildung nicht gelingt. ${ }^{22}$ Eine ausgeprägte Bildungsrationierung auf Sekundarstufe I scheint demnach die Bildungschancen der Schulentlassenen merklich zu beeinträchtigen - wohl auch über die erste Schwelle hinaus.

Die Bedeutung des räumlich-institutionellen Kontexts lässt sich ferner auch am Effekt der Sprachregion ablesen. So ist in der italienischsprachigen Schweiz die Chance markant höher als in der deutschsprachigen, direkt anstatt via Brückenangebot in eine Sek.-II-Ausbildung einzusteigen. Dies hat massgeblich damit zu tun, dass solche Angebote im Übergangssystem der italienischen Schweiz institutionell kaum verankert sind. In der Romandie ist innerhalb der Zwischenlösungen der Anteil der 10. Schuljahre deutlich höher als in der Deutschschweiz.

Zusammenfassend spricht die Modellierung des Übergangs an der ersten Schwelle dafür, dass vor allem der absolvierte Sek.-I-Schultyp, das Geschlecht und die soziale Herkunft sowie der räumlich-institutionelle Kontext wichtig sind. Bei manchen Jugendlichen spielen zudem berufliche Orientierungsschwierigkeiten eine Rolle, wogegen individuelle Defizite bezüglich Schulleistungen, kognitiver und nicht-kognitiver Kompetenzen und Motivationen wenig erklären. Der verbreitete

$21 \quad$ Marginaler Effekt, wenn alle anderen Prädiktoren auf ihrem Mittelwert fixiert sind.

22 Theoretisch ist zu erwarten, dass davon ausschliesslich Schulentlassene betroffen sind, die faktisch einen Schultyp mit "Grundansprüchen» absolviert haben und die je nach kantonalem Sek.-ISystem unterschiedlich zahlreich auf den regionalen Lehrstellenmarkt gelangen. Ein entsprechender Interaktionseffekt zwischen dem individuell absolvierten Schultyp und der kantonalen "RealschulQuote» lässt sich mit den verfügbaren Daten nicht robust schätzen, stützt aber zumindest der Tendenz nach eine solche Interpretation $(\mathrm{Bz}=\mathrm{B} \ddot{\mathrm{u}}=\mathrm{Bo} \approx 2.4, \mathrm{p}=0.081)$. 
Übertritt in Brückenangebote kann somit nicht auf entsprechende individuelle Defizite zurückgeführt werden.

\subsubsection{Wirkungen unterschiedlicher Übergänge auf die nachobligatorische Ausbildung}

Die Wirkung des Übertritts in ein Brückenangebot sowie des Verbleibs ohne Anschlusslösung schätzen wir wie in Abschnitt 4.2 beschrieben mittels PropensityScore-Matching. Tabelle 3 enthält die Populationsschätzer für die mittleren kausalen Effekte (ATT) auf die Gruppen von Schulentlassenen, die einen gegebenen Übergang ("Treatment") durchlaufen - jeweils im Vergleich zu einem der anderen Übergänge. Wir weisen separate ATT-Schätzungen für die Wahrscheinlichkeiten aus, dass die Jugendlichen zwei Jahre später eine solche Ausbildung besuchen, respektive dass sie sechs Jahre später eine solche Ausbildung erfolgreich abgeschlossen haben. Für beide Wirkungsdimensionen stellen wir Ergebnisse auf Basis zweier unterschiedlicher Gewichtungen dar: Einer reinen Designgewichtung, welche die individuell unterschiedlichen Auswahlwahrscheinlichkeiten kompensiert, und einer Gewichtung, die zusätzlich auch die Panel-Attrition korrigiert (vgl. Abschnitt 4.2).

\section{Tabelle 3 Geschätzter durchschnittlicher Kausaleffekt (ATT)}

\begin{tabular}{|c|c|c|c|c|c|c|c|c|}
\hline \multirow{3}{*}{$\begin{array}{l}\text { Wirkungsdimension: } \\
\text { Stichprobengewicht: } \\
\text { Kennwert: }\end{array}$} & \multicolumn{4}{|c|}{$\begin{array}{r}\text { Besuch einer Sek.-II-Ausbildung im 2. Jahr } \\
\text { nach Ende der oblig. Schule }\end{array}$} & \multicolumn{4}{|c|}{$\begin{array}{r}\text { Abschluss einer Sek.-II-Ausbildung bis zum } \\
6 \text {. Jahr nach Ende der oblig. Schule }\end{array}$} \\
\hline & \multicolumn{2}{|c|}{ Designgewichtung } & \multicolumn{2}{|c|}{ Design \& Attrition } & \multicolumn{2}{|c|}{ Designgewichtung } & \multicolumn{2}{|c|}{ Design \& Attrition } \\
\hline & ATT & (SE) & ATT & (SE) & ATT & (SE) & ATT & (SE) \\
\hline \multicolumn{9}{|c|}{ Referenzkategorie: Direkter Einstieg in eine Sek.-II-Ausbildung } \\
\hline 10. Schuljahr & -0.22 & $(0.031)^{* * *}$ & -0.24 & $(0.053)^{* * *}$ & -0.12 & $(0.033)^{* * *}$ & -0.17 & $(0.043)^{* * *}$ \\
\hline Andere Zwischenlösung & -0.25 & $(0.036)^{* * *}$ & -0.24 & $(0.030)^{* * *}$ & -0.18 & $(0.034)^{* * *}$ & -0.14 & $(0.043)^{* *}$ \\
\hline Ohne Anschlusslösung & -0.36 & $(0.058)^{* * *}$ & -0.35 & $(0.054)^{* * *}$ & -0.30 & $(0.079)^{* * *}$ & -0.56 & $(0.196)^{* *}$ \\
\hline \multicolumn{9}{|c|}{ Referenzkategorie: Ohne Anschlusslösung } \\
\hline 10. Schuljahr & 0.15 & $(0.064)^{*}$ & 0.11 & $(0.079)$ & 0.18 & $(0.085)^{*}$ & 0.39 & $(0.194)^{*}$ \\
\hline Andere Übergangslösung & 0.11 & $(0.066)^{+}$ & 0.12 & $(0.061)^{+}$ & 0.13 & $(0.086)$ & 0.43 & $(0.196)^{*}$ \\
\hline \multicolumn{9}{|c|}{ Referenzkategorie: Andere Übergangslösung } \\
\hline 10. Schuljahr & 0.03 & $(0.047)$ & -0.01 & $(0.062)$ & 0.06 & $(0.047)$ & -0.03 & $(0.060)$ \\
\hline
\end{tabular}

Anmerkungen: SE: De-Luna-Bootstrap Standardfehler, 1000 Replikationen; Signifikanzniveaus: ${ }^{* * *} p \leq 0.001 ;{ }^{* *} p \leq 0.01$; ${ }^{*} p \leq 0.05 ;{ }^{+} p \leq 0.10$.

Quelle: TREE, eigene Berechnungen.

Wir betrachten zunächst die Ergebnisse im oberen Teil der Tabelle 3, in dem der Übergang via Brückenangebote sowie der Verbleib ohne Anschlusslösung mit dem direkten Einstieg in eine zertifizierende Ausbildung verglichen werden. Die betreffenden Ergebnisse stützen zunächst eindrücklich unsere erste wirkungsbezogene $\mathrm{Hy}$ pothese, wonach die nachobligatorischen Ausbildungschancen von Schulentlassenen 
nachhaltig beeinträchtigt werden, wenn sie nicht direkt in eine anerkannte Sek.II-Ausbildung eintreten. So liegt die Chance, dass Schulentlassene mit identischen individuellen, schulischen, familiären und kontextuellen Ausgangsbedingungen zwei Jahre nach Erfüllung der Schulpflicht eine solche Ausbildung besuchen, um 22 bis 25 Prozent tiefer (ATT-0.22 / -0.25), wenn sie zunächst ein Brückenangebot absolvieren, statt direkt in eine zertifizierende Ausbildung überzutreten. Während rund 96 Prozent der Direkteinsteiger mit vergleichbaren Voraussetzungen zu diesem Zeitpunkt eine solche Ausbildung besuchen, sind es bei den Absolventen von Brückenangeboten lediglich zwischen 71 und 74 Prozent. Auch die Chance, eine zertifizierende Sek.-II-Ausbildung abzuschliessen, ist erheblich beeinträchtigt: Sie liegt um 12 (10. Schuljahr) respektive 18 Prozent (andere Übergangslösung) tiefer als bei den Direkteinsteigern mit vergleichbaren Voraussetzungen, die zu 93 Prozent eine Sek.-II-Ausbildung abschliessen. Dies spricht für eine anhaltende Wirkung des verzögerten Einstiegs, welche nicht oder nur teilweise durch spätere Prozesse kompensiert wird. Verbleiben Schulentlassene allerdings ganz ohne Anschlusslösung, so beeinträchtigt dies ihre Chancen noch weit stärker: Bezüglich des Einstiegs reduzieren sie sich um zirka 35 und bezüglich des Abschlusses um 30 Prozent. Unter Berücksichtigung der Nonresponse-Korrektur beträgt das Chancen-Minus gar rund 56 Prozent. Eine substanzielle Unterschätzung des Effekts bei Verzicht auf eine Nonresponse-Korrektur ist dabei plausibel, da die Jugendlichen mit dem grössten Drop-Out-Risiko nachweislich auch überproportional häufig aus dem Panel herausfallen (vgl. Sacchi 2011; Sacchi et al. 2011). Davon abgesehen sind die geschätzten mittleren Kausaleffekte für beide Gewichtungen ähnlich gross. Sämtliche ATTs zum Vergleich des Direkteinstiegs mit den anderen Übergängen sind zudem unabhängig von der verwendeten Gewichtung hoch signifikant.

Unsere zweite Wirkungshypothese postuliert, dass der Besuch eines Brückenangebots die negativen Folgen eines verzögerten Einstiegs teilweise zu kompensieren vermag. Auch diese Hypothese wird durch unsere Ergebnisse gestützt. So zeigt der zweite Abschnitt von Tabelle 3 zum Vergleich der Brückenangebote mit dem Verbleib ohne Anschlusslösung, dass die betreffenden ATTs durchwegs ein positives Vorzeichen aufweisen, wobei sie statistisch allerdings nicht durchwegs gesichert sind. Dank dem Besuch eines Brückenangebots erhöht sich im Vergleich zur Gruppe ohne Anschlusslösung demnach zumindest tendenziell die Chance, innert zwei Jahren in eine Sek.-II-Ausbildung einzutreten (um 11 bis 15\%) und auch die Chance, diese später erfolgreich abzuschliessen. Der letztgenannte Effekt ist dabei unter Berücksichtigung der Nonresponse-Korrektur mit 39 bis 43 Prozent sehr bedeutend und auf konventionellem Niveau signifikant, während er ansonsten mit 13 bis 18 Prozent deutlich bescheidener ausfällt - und im Fall der anderen Übergangslösungen statistisch nicht gesichert ist. Insgesamt stützen die Ergebnisse die Vermutung, dass Brückenangebote eine positive Wirkung entfalten, welche allerdings die Folgen eines verzögerten Einstiegs nicht voll zu kompensieren vermag. Vielmehr bleiben 
die Chancen von Schulentlassenen ohne direkten Einstieg auch dann nachhaltig beeinträchtigt, wenn sie ein Brückenangebot absolvieren. Dies zeigt der Vergleich mit den direkt einsteigenden Jugendlichen im oberen Teil der Tabelle.

Keine Unterstützung in den Daten findet unsere dritte Wirkungshypothese, die dem institutionell vergleichsweise gut verankerten 10. Schuljahr eine stärkere kompensatorische Wirkung zuschreibt als den anderen Übergangslösungen. Die betreffenden Effektdifferenzen im unteren Teil der Tabelle sind klein und statistisch nicht gesichert.

$\mathrm{Zu}$ diesen Interpretationen dieses Abschnitts ist grundsätzlich anzumerken, dass sich in den beobachteten Treatment-Effekten verschiedene Prozesse vermischen: Dazu zählen etwa die Suche nach einem Ausbildungsplatz nach einem Überbrückungsjahr, der Abbruch einer begonnenen Ausbildung (sei es im Anschluss an einen Direkteinstieg oder an ein Brückenangebot) oder der spätere Wiedereinstieg nach einem Ausbildungsabbruch. Solche intermediären, im beobachteten Durchschnittseffekt vermengten Prozesse verdienten zweifellos eine separate Analyse, was allerdings den Rahmen dieses Beitrags deutlich gesprengt hätte.

\section{Schlussfolgerungen}

Unsere Ergebnisse zu den Selektionsprozessen beim Übergang in Brückenangebote an der ersten Schwelle legen den Schluss nahe, dass dafür in erster Linie die soziale Herkunft, der absolvierte Sek.-I-Schultyp, berufliche Orientierungsschwierigkeiten, die Unterstützung durch Schule und Elternhaus, die räumlich-institutionellen Kontextbedingungen sowie das Geschlecht relevant sind. Nicht-kognitive Kompetenzen und motivationale Aspekte sind nur am Rande relevant, während sich für die Schulnoten und kognitive Kompetenzen (PISA literacy) keinerlei eigenständiger Einfluss nachweisen lässt. Die Befunde stehen damit in markantem Gegensatz zum individualisierenden bildungspolitischen Diskurs, der die Gründe für den Übertritt in ein Brückenangebot in erster Linie in Kompetenzdefiziten, ungünstigen Dispositionen und ungenügenden schulischen Leistungen der einzelnen Jugendlichen ortet. Gemäss unserer Analyse ist dies höchstens am Rande der Fall. Massgeblich sind vielmehr vor allem Herkunfts- und Strukturmerkmale, welche die Jugendlichen nicht beeinflussen können.

Mit Blick auf die drei von Meyer (2003) postulierten Funktionen von Zwischenlösungen legen die dargestellten Ergebnisse den Schluss nahe, dass die Kompensationsfunktion - entgegen ihrer prominenten Rolle im bildungspolitischen Diskurs, aber in Übereinstimmung mit den Ergebnissen von Bayard (2011) - de facto für die beobachteten Übergangs- bzw. Selektionsprozesse keine bedeutende Rolle spielt. Mit der Orientierungs- $b z w$. Berufswahl-Funktion stehen die Ergebnisse dagegen besser in Einklang, treten Jugendliche ohne klare Ausbildungs- und Berufspläne doch gehäuft 
in Brückenangebote ein. Dies betrifft allerdings nur einen relativ kleinen Teil der Schulentlassenen. Zur systemischen Pufferfunktion lassen sich aus unseren Ergebnissen keine direkten Aussagen ableiten. Dafür wären Kohortenvergleiche nötig, im Rahmen welcher die Variation der dafür massgeblichen Angebots-Nachfrage-Relation auf dem Lehrstellenmarkt kontrolliert werden kann. Vollauf bestätigt wird jedoch die Erwartung, wonach in einer Phase starken Nachfrage-Überhangs auf dem Lehrstellenmarkt bei der Selektion in Brückenangebote vor allem der Sek.-I-Schultyp, askriptive Merkmale (insbesondere die soziale Herkunft und das Geschlecht) sowie institutionelle Rahmenbedingungen wirksam werden. Das Abpuffern des Mangels an zertifizierenden Ausbildungsplätzen mittels Brückenangeboten erfolgt somit sozial äusserst selektiv. Hingegen finden sich keine Hinweise darauf, dass die Selektion dabei auch leistungsbezogen bzw. anhand meritokratischer Prinzipien erfolgt.

Die Analyse der Wirkungen liefert starke Indizien, wonach sich die Chance, in eine zertifizierende Sek-II-Ausbildung einzutreten und diese erfolgreich abzuschliessen, substanziell reduziert, wenn Schulentlassene nicht direkt in eine solche Ausbildung eintreten. Im Vergleich zu direkt einsteigenden Jugendlichen verringert sich dadurch die Chance, einen Sek.-II-Abschluss zu erlangen, um 12 bis 18 Prozent. Allerdings reduziert sie sich sogar um 30 bis 56 Prozent, wenn Jugendliche nach der obligatorischen Schule zunächst ganz ohne Anschlusslösung verbleiben. Brückenangebote entfalten demnach durchaus eine positive Wirkung auf die Abschlusschancen, welche aber die negative Wirkung des verzögerten Einstiegs nicht vollständig zu kompensieren vermag. Anhaltspunkte für Unterschiede zwischen dem 10. Schuljahr und anderen Arten von Übergangslösungen finden wir dabei nicht. Vielmehr bleiben die Chancen eines Sek.-II-Abschlusses unabhängig von der Art des absolvierten Brückenangebots erheblich beeinträchtigt.

Aus bildungspolitischer Sicht sind diese Befunde in doppelter Hinsicht brisant: Zum einen sind sie schwer mit der Annahme vereinbar, dass die Chancengerechtigkeit beim Übertritt in Brückenangebote gewahrt ist. Zum anderen liefern sie auch starke Evidenz dafür, dass die sozial selektiven, unter meritokratischen Gesichtspunkten hingegen weitgehend zufälligen Verzögerungen beim Einstieg in eine Sek.-II-Ausbildung die Bildungschancen der Betroffenen substanziell und nachhaltig beeinträchtigen. Angesichts des hohen Anteils an Jugendlichen in Brückenangeboten lässt sich somit feststellen, dass die institutionelle Ausgestaltung des Übergangssystems an der ersten Schwelle dem bildungspolitisch konsensualen Ziel einer Erhöhung der Sek.-II-Abschlussquote direkt zuwider läuft. Mit Blick auf dieses Ziel wäre es aufgrund unserer Befunde essenziell, Brückenangebote als systemischen Puffer so weit wie möglich zu reduzieren, indem ein stabiles und ausreichendes Angebot an zertifizierenden Ausbildungsplätzen auf Sekundarstufe II sichergestellt wird. Idealerweise wäre dafür ein gewisser Angebots-Überhang erforderlich (Zihlmann 2004). Wenn eine Beeinträchtigung der nachobligatorischen Bildungschancen schulentlassener Jugendlicher vermieden werden soll, müsste bei 
Lehrstellenknappheit eine ausreichende Zahl von zusätzlichen zertifizierenden Ausbildungsplätzen geschaffen werden - im Bedarfsfall auch ausserhalb des marktförmig verfassten Teils des Lehrstellenangebots (z. B. Lehrwerkstätten).

Auch wenn die Ergebnisse bezüglich dieser zentralen Folgerung unseres Erachtens nur wenig Interpretationsspielraum lassen, möchten wir auch auf die Grenzen unserer Analyse hinweisen. Erstens fehlt ein direkter Test der postulierten Kausalmechanismen. Es bleibt somit letztlich offen, inwieweit die beobachteten Wirkungen eines verzögerten Einstiegs wie postuliert auf den psychosozialen Folgen einer vergeblichen Lehrstellensuche sowie auf statistischen Diskriminierungen beruhen. Ebenso bleibt offen, worauf die kompensierende Funktion der Brückenangebote beruht und welche intervenierenden Prozesse allenfalls im späteren Ausbildungsverlauf zusätzlich ins Spiel kommen. Hier bedarf es weiterer Forschung, um intervenierende Prozesse und kausale Mechanismen zu identifizieren. Grundsätzlich ist unsere kausale Interpretation der beobachteten Unterschiede aufgrund der aussergewöhnlich umfassenden Kontrolle der massgeblichen Selektionsprozesse aber relativ gut abgesichert.

Zweitens beziehen sich unsere Analysen auf die Schulentlassenen-Kohorte des Jahres 2000. Es stellt sich daher die Frage, inwieweit die dargestellten Ergebnisse unter heutigen Bedingungen weiterhin Gültigkeit behalten. Im öffentlichen Diskurs herrscht die Meinung vor, dass sich die Lehrstellenknappheit und damit die Warteschlange in den kommenden Jahren aus demografischen Gründen weiter reduzieren werden. Wir vermuten allerdings, dass sich dadurch primär die Zahl der Betroffenen verringert, wogegen die soziale Selektivität beim Übergang in Brückenangebote und die Beeinträchtigung der Bildungschancen im Falle eines verzögerten Einstiegs davon unberührt bleiben. Deren stigmatisierende Wirkung könnte sich im Gegenteil sogar verstärken, wenn die Zahl der Betroffenen sinkt. Mit der bevorstehenden Lancierung einer zweiten TREE-Schulabgänger-Kohorte wird sich Gelegenheit bieten, dies empirisch zu prüfen.

\section{Literaturverzeichnis}

Abadie, Alberto und Guido W. Imbens. 2008. On the failure of the bootstrap for matching estimators. Econometrica 76(6): 1537-1557.

Acock, Alan C. 2005. Working with missing values. Journal of Marriage and the Familiy 67(4): 1012-1028.

Austin, Peter C. 2011. An introduction to propensity score methods for reducing the effects of confounding in observational studies. Multivariate Behavioral Research 46(3): 399-424.

Babel, Jacques, Laurent Gaillard und Pascal Strübi. 2013. Bildungsperspektiven. Szenarien 2013-2022 für das Bildungssystem. Neuenburg: Bundesamt für Statistik.

Bauer, Phillip und Regina T. Riphahn. 2006. Timing of school tracking as a determinant of intergenerational transmission of education. Economics Letters 91(1): 90-97. 
Bauer, Phillip und Regina T. Riphahn. 2007. Intergenerational transmission of educational attainment: Evidence from Switzerland on natives and second generation immigrants. Journal of Population Economics 20(1): 121-148.

Bayard, Sybille. 2011. Berufslehre, schulische Ausbildung oder Zwischenlösung? Die Bedeutung sozialer Faktoren und nichtkognitiver Kompetenzen beim Übertritt an der ersten Schwelle. Dissertation, Philosophische Fakultät, Universität Zürich.

BBT (Bundesamt für Berufsbildung und Technologie). 2000. BBT-Empfehlungen 2000 für Brückenangebote zwischen obligatorischer Schule und Berufsbildung. Bern: Bundesamt für Berufsbildung und Technologie.

BBT (Bundesamt für Berufsbildung und Technologie) (Hrsg.). 2006. Lehrstellenbarometer August 2006. Detaillierter Ergebnisbericht zur Umfrage bei Jugendlichen und Unternehmen. Bern: Bundesamt für Berufsbildung und Technologie.

Beicht, Ursula. 2009. Verbesserung der Ausbildungschancen oder sinnlose Warteschleife? Zur Bedeutung und Wirksamkeit von Bildungsgängen am Übergang Schule - Berufsausbildung. Bielefeld: BIBB.

Bell, David N.F. und David G. Blanchflower. 2010. Youth unemployment: Déjà vu? IZA Discussion Paper 4705. Bonn: Forschungsinstitut zur Zukunft der Arbeit.

BFS/TREE (Hrsg.). 2003. Wege in die nachobligatorische Ausbildung. Die ersten zwei Jahre nach Austritt aus der obligatorischen Schule. Reihe "Bildungsmonitoring Schweiz». Neuenburg: Bundesamt für Statistik.

BIBB (Bundesinstitut für Berufsbildung). 2009. Berufsbildungsbericht 2009-Informationen und Analysen zur Entwicklung der beruflichen Bildung. Bonn: BIBB.

Brookhart, M. Alan, Sebastian Schneeweiss, Kenneth J. Rothman, Robert J. Glynn, Jerry Avorn und Til Stürmer. 2006. Variable selection for propensity score models. American Journal of Epidemiology 163(12): 1149-1156.

Clark, Andrew E., Yannis Georgellis und Peter Sanfey. 2001. Scarring: The psychological impact of past unemployment. Economia 68(270): 221-241.

de Luna, Xavier, Per Johansson und Sara Sjöstedt-de Luna. 2010. Bootstrap Inference for K-nearest Neighbour Matching Estimators. Discussion Paper Series 5361. Forschungsinstitut zur Zukunft der Arbeit, Bonn.

DuGoff, Eva H., Megan Schuler und Elizabeth A. Stuart. 2014. Generalizing observational study results: Applying propensity score methods to complex surveys. Health Services Research 49(1): 284-303.

Egger, Dreher \& Partner. 2007. Vertiefungsstudie: Bildungsangebote im Übergang von der obligatorischen Schule in die Berufsbildung. Erstellt im Auftrag des Bundesamtes für Berufsbildung und Technologie. Bern: ED\&P.

Falter, Jean-Marc und Florian Wendelspiess Chávez Juárez. 2011. Does tracking shape intergenerational transmission of educational attainment? Evidence from Switzerland. Labor: Human Capital eJournal 10: 1-15.

Furnham, Adrian. 1985. Youth unemployment: A review of the literature. Journal of Adolescence 8(2): 109-124.

Gangl, Markus. 2010. Causal inference in sociological research. Annual Review of Sociology 36: 21-47.

Gangl, Markus und Thomas A. DiPrete. 2004. Kausalanalyse durch Matchingverfahren. S. 396-420 in Methoden der Sozialforschung. Bd. Sonderheft 44, hrsg. von Andreas Diekmann. Opladen: Westdeutscher Verlag.

Gertsch, Marianne, Alexander Gerlings und Caterina Modetta. 1999. Der Lehrstellenbeschluss. Evaluation. Studie über Brückenangebote. Bern: Koordinationsstelle für Weiterbildung der Universität Bern.

Haeberlin, Urs, Christian Imdorf und Winfried Kronig. 2004. Chancenungleichheit bei der Lehrstellensuche. Der Einfluss von Schule, Herkunft und Geschlecht. Bern: Nationales Forschungsprogramm «Bildung und Beschäftigung» (NFPNR 43). 
Helland, Håvard und Liv Anne Støren. 2006. Vocational education and the allocation of apprenticeships: Equal chances for applicants regardless of immigrant background? European Sociological Review 22(3): 339-351.

Henkel, Linda A. und Mara Mather. 2007. Memory attributions for choices: How beliefs shape our memories. Journal of Memory and Language 57(2): 163-176.

Herzog, Walter, Markus P. Neuenschwander und Evelyne Wannack. 2004. In engen Bahnen: Berufswahlprozess bei Jugendlichen. Bern: Nationales Forschungsprogramm «Bildung und Beschäftigung» (NFPNR 43).

Herzog, Walter, Markus P. Neuenschwander und Evelyne Wannack. 2006. Berufswahlprozess. Wie sich Jugendliche auf ihren Beruf vorbereiten. Bern: Haupt.

Hosmer, David W. und Stanley Lemeshow. 2000 [1989]. Applied Logistic Regression. New York: John Wiley \& Sons.

Hupka, Sandra, Stefan Sacchi und Barbara Stalder. 2010. Social origin and the access to upper secondary education in Switzerland: A comparison of company-based apprenticeship and exclusively school based programmes. Schweizerische Zeitschrift für Soziologie 36(1): 11-31.

Hupka-Brunner, Sandra, Thomas Meyer, Barbara E. Stalder und Anita Keller. 2011. PISA-Kompetenzen und Übergangswege: Ergebnisse aus der Schweizer TREE-Studie. S. 175-190 in Neue Jugend? Neue Ausbildung? Beiträge aus der Jugend- und Bildungsforschung, hrsg. von BIBB/DJI. Bonn: Bundesinstitut für Berufsbildung.

Imdorf, Christian. 2007. Lehrlingsselektion in KMU. Nationales Forschungsprogramm «Integration und Ausschluss» (NFPNR 51). Kurzbericht März 2007. Freiburg: Heilpädagogisches Institut der Universität Freiburg.

Keller, Anita, Sandra Hupka-Brunner und Thomas Meyer. 2010. Nachobligatorische Ausbildungsverläufe in der Schweiz: Die ersten sieben Jahre. Ergebnisübersicht des Jugendlängsschnitts TREE, Update 2010. Basel: TREE, Universität Basel.

Kerr, Sari Pekkala, Tuomas Pekkarinen und Roope Uusitalo. 2013. School tracking and development of cognitive skills. Journal of Labor Economics 31(3): 577-602.

Leuven, Edwin und Barbara Sianesi. 2003. PSMATCH2: Stata Module to Perform Full Mahalanobis and Propensity Score Matching, Common Support Graphing, and Covariate Imbalance Testing. Boston: Boston College Department of Economics, https://ideas.repec.org/c/boc/bocode/s432001.html.

Meyer, Thomas. 2003. Zwischenlösung - Notlösung? S. 101-109 in Wege in die nachobligatorische Ausbildung. Die ersten zwei Jahre nach Austritt aus der obligatorischen Schule, hrsg. von BFS/TREE. Neuenburg: Bundesamt für Statistik.

Meyer, Thomas. 2009. Wer hat, dem wird gegeben: Bildungsungleichheit in der Schweiz. S. 60-81 in Sozialbericht 2008, hrsg. von Christian Suter, Silvia Perrenoud, René Levy, Ursina Kuhn, Dominique Joye und Pascale Gazareth. Zürich: Seismo Verlag.

Meyer, Thomas und Kathrin Bertschy. 2011. The long and winding road from education to labour market. S. 92-119 in Transitionsprozesse im Jugendalter: Ergebnisse der Schweizer Längsschnittstudie TREE, hrsg. von Manfred Max Bergmann, Sandra Hupka-Brunner, Anita Keller, Thomas Meyer und Barbara E. Stalder. Zürich: Seismo Verlag.

Meyrat, Michael. 2004. Lehrstellenbeschluss 2. Schlussbericht der Evaluation. Bern: Bundesamt für Berufsbildung und Technologie; Koordinationsstelle für Weiterbildung der Universität Bern.

Müller, Barbara und Stefan C. Wolter. 2014. The role of hard-to-obtain information on ability for the school-to-work transition. Empirical Economics 46(4): 1447-1471.

Neuenschwander, Markus P. 2006. Entwicklungsanlass oder Entwicklungshemmer? Schulübergänge und Selbstkonzepte im Jugendalter. Akzente 3/06: 9-11.

Neuenschwander, Markus P. 2008. Elternunterstützung im Berufswahlprozess. S. 135-154 in Berufliche Übergänge: Psychologische Grundlagen der Berufs-, Studien-und Laufbahnberatung, hrsg von Damian Länge und Andreas Hirschi. Zürich: LIT-Verlag. 
Niederberger, Josef Martin. 2003. Brückenangebote: Struktur und Funktion. Die Rolle von Geschlecht und Nationalität. Unter Mitarbeit von Christine Ackermann. Neuenburg: Swiss Forum for Migration and Population Studies.

Pfeffer, Fabian. 2008. Persistent inequality in educational attainment and its institutional context. European Sociological Review 24. Advance access published online on May 1, 2008, doi:10.1093/esr/jen026.

Royston, Patrick. 2004. Multiple imputation of missing values. The Stata Journal 4(3): 227-241.

Royston, Patrik. 2007. Multiple imputation of missing values: Further Update of ICE, with an Emphasis on Interval Censoring. The Stata Journal 7(4): 445-464.

Rubin, Donald B. 1987. Multiple Imputation for Non-response in Surveys. New York: John Wiley \& Sons.

Rubin, Donald B. 1996. Multiple imputation after 18+ years. Journal of the American Statistical Association 91(434): 473-489.

Sacchi, Stefan. 2011. TREE-Längsschnittgewichtung: Konstruktion und Anwendung. Dokumentation zu den Erhebungswellen 2000 bis 2010. Bern und Zürich: TREE und Cue Sozialforschung.

Sacchi, Stefan, Sandra Hupka-Brunner, Barbara E. Stalder und Markus Gangl. 2011. Die Bedeutung von sozialer Herkunft und Migrationshintergrund für den Übertritt in anerkannte nachobligatorische Ausbildungen in der Schweiz. S. 120-156 in Transitionen im Jugendalter. Ergebnisse der Schweizer Längsschnittstudie TREE, hrsg. von Manfred Max Bergmann, Sandra Hupka-Brunner, Anita Keller, Thomas Meyer und Barbara E. Stalder. Zürich: Seismo.

SBFI (Staatssekretariat für Bildung, Forschung und Innovation) (Hrsg.). 2014. Lehrstellenbarometer August 2014. Detaillierter Ergebnisbericht. Luzern: LINK Institut für Markt- und Sozialforschung.

Scarpetta, Stefano, Anne Sonnet und Thomas Manfredi. 2010. Rising youth unemployment during the crisis: How to prevent negative long-term consequences on a generation? OECD Social, Employment and Migration Papers 106. Paris: OECD.

Schweizerische Koordinationsstelle für Bildungsforschung (Hrsg.). 2014. Bildungsbericht 2014. Aarau: SKBF.

Solga, Heike. 2005. Ohne Abschluss in die Bildungsgesellschaft. Die Erwerbschancen gering qualifizierter Personen aus ökonomischer und soziologischer Perspektive. Opladen: Verlag Barbara Budrich.

Solga, Heike und Rolf Becker. 2012. Soziologische Bildungsforschung - eine kritische Bestandsaufnahme. Kölner Zeitschrift für Soziologie und Sozialpsychologie Sonderheft 52: 7-41.

Stalder, Barbara E., Thomas Meyer und Sandra Hupka-Brunner. 2011. TREE Project Documentation. S. 66-85 in Transitionen im Jugendalter: Ergebnisse der Schweizer Längsschnittstudie TREE, hrsg. von Manfred Max Bergmann, Sandra Hupka-Brunner, Anita Keller, Thomas Meyer und Barbara E. Stalder. Zürich: Seismo Verlag.

Stuart, Elizabeth A. 2010. Matching methods for causal inference: A review and a look forward. Statistical Science 25(1): 1-21.

Tondorf, Anne und Fabiana Schuppli. 2012. Der Übergang von einer schulischen Zwischenlösung in die berufliche Grundbildung. Lizentiatsarbeit, Universität Zürich.

Ulrich, Joachim Gerd. 2013. Institutionelle Mechanismen der (Re-)Produktion von Ausbildungslosigkeit. S. 93-106 in Prozesse sozialer Ungleichheit. Bd. 40, hrsg. von Susanne Siebholz, Edina Schneider, Anne Schippling, Susann Busse und Sabine Sandring. Wiesbaden: Springer Fachmedien.

Vandenberghe, Vincent (Hrsg.). 2010. The Long-term Effects of Recessions on Youth. Reviewing the Evidence. Louvain-la-Neuve : Université catholique de Louvain (UCL).

Winship, Christopher und Stephen L. Morgan. 1999. The estimation of causal effects from observational data. Annual Review of Sociology 25: 659-706.

Zihlmann, René. 30.07.2004. Es wäre ein Überhang an Lehrstellen nötig. Voraussetzungen für einen funktionierenden Markt. S. 47 in Neue Zürcher Zeitung. Zürich: NZZ. 


\section{$7 \quad$ Anhang}

Tabelle A1 Verwendete Prädiktoren und ungewichtete Verteilungskennwerte

\begin{tabular}{|c|c|c|c|}
\hline \multirow[t]{2}{*}{ Einbezogene Prädiktoren } & \multicolumn{2}{|c|}{ Kategoriale Variablen } & Skalen \\
\hline & Ausprägungen & $\begin{array}{l}\text { Verteilung Mittelwert } \\
(\%)\end{array}$ & $\begin{array}{l}\text { Standard- } \\
\text { abweichung }\end{array}$ \\
\hline
\end{tabular}

Schulleistungen, Kompetenzen, Motivationen

Sprachnote (st41q04) [5\%]

Mathematiknote (st41q05) [5\%]

PISA-Lesekompetenz (wleread) [0\%]

PISA mathem. Kompetenz (wlemath) [45\%]

Verbales Selbstkonzept (scverb) [2\%]

Mathemat. Selbstkonzept (matcon) [3\%]

Selbstwirksamkeit (selfef) [1\%]

Kontrollüberzeugung (cexp) [2\%]

Persistenz (effper) [2\%]

Instrumentelle Motivation (insmot) [1\%]

Fehlzeiten (st29q01) [1\%]

$\begin{array}{lr}\text { "Above" (Ref.) } & 69 \\ \text { "At the mark" } & 26 \\ \text { "Below" } & 5 \\ \text { "Above" (Ref.) } & 62 \\ \text { "At the mark" } & 26 \\ \text { "Below" } & 12\end{array}$

69

26

5

62

26

12

\begin{tabular}{ll}
492 & 81 \\
519 & \multicolumn{1}{l}{84} \\
-0.01 & 0.89 \\
0.04 & 0.99 \\
-0.02 & 0.85 \\
-0.05 & 0.90 \\
-0.06 & 0.95 \\
-0.03 & 0.87
\end{tabular}

Keine (Ref.) $\quad 66$

Eine bis zwei $\quad 25$

Mehr als zwei 8

Ausbildungspläne und Laufbahnorientierung

Beruf, Berufsstatus mit 30 (st40q01, bhtr) [0\%] Keine Antwort

Weiss Nicht 3

Status 1-3. Quartil $\quad 80$

(Ref.)

Status 4. Quartil 16

Nachobl. Ausbildungspläne (st42q01) [10\%] Weiss nicht 2

Soziale Selektivität

Schultyp Sekundarstufe I (typ) [0\%] $\quad$ Progymnasialer Typ 20

Erw. Ansprüche (Ref.) $\quad 37$

Grundansprüche 27

Integrierter Typ $\quad 17$

Geschlecht (sex) [0\%] Weiblich $\quad$ W4 
Fortsetzung der Tabelle A1.

\begin{tabular}{|c|c|c|c|c|}
\hline \multirow[t]{2}{*}{ Einbezogene Prädiktoren } & \multicolumn{2}{|c|}{ Kategoriale Variablen } & \multicolumn{2}{|c|}{ Skalen } \\
\hline & Ausprägungen & $\begin{array}{r}\text { Verteilung } \\
(\%)\end{array}$ & Mittelwert & $\begin{array}{l}\text { Standard- } \\
\text { abweichung }\end{array}$ \\
\hline \multirow[t]{3}{*}{ Ausbildungsniveau Eltern [4\%] } & Ohne nachobl. Ausb. & 11 & & \\
\hline & Mit Sek.-II-Ausb. (Ref.) & 48 & & \\
\hline & mit Tertiärausbildung & 40 & & \\
\hline Finanz. Ressourcen Familie (wealth) [0\%] & & & 0.03 & 0.81 \\
\hline Status Herkunftsfamilie (isei) [5\%] & & & 42 & 15 \\
\hline \multirow[t]{5}{*}{ Geburtsland des Vaters (st16n03) [1\%] } & Schweiz (Ref.) & 71 & & \\
\hline & $D, F, B, N L$ & 3 & & \\
\hline & Italien, Spanien & 10 & & \\
\hline & P, Balkanländer, Türkei & 12 & & \\
\hline & Übrige Länder & 4 & & \\
\hline Unterstützung Familie (famedsup) [1\%] & & & 0.01 & 0.96 \\
\hline Unterstützung Lehrer (teachsup) [0\%] & & & 0.07 & 0.94 \\
\hline Eltern: Kulturelles Interesse (cultcom) [1\%] & & & -0.13 & 0.90 \\
\hline Eltern: Soziales Interesse (soccom) [1\%] & & & 0.01 & 0.96 \\
\hline \multicolumn{5}{|l|}{ Räumlich-institutioneller Kontext } \\
\hline $\begin{array}{l}\text { Kantonaler Anteil des Sek.-I-Schultyps mit } \\
\text { «Grundansprüchen» }[0 \%]\end{array}$ & & & 0.28 & 0.10 \\
\hline \multirow[t]{3}{*}{ Sprachregion (reg_ling) [0\%] } & Deutschschweiz (Ref.) & 54 & & \\
\hline & Romandie & 35 & & \\
\hline & Tessin & 11 & & \\
\hline \multirow[t]{2}{*}{ Siedlungstyp (agglo) [0\%] } & Stadt & 39 & & \\
\hline & Agglomeration / Land & 61 & & \\
\hline
\end{tabular}

Anmerkungen: Stichprobegrösse: $n=3605$. Neben dem Variablenlabel sind in Klammern die originalen PISAVariablenbezeichnungen (soweit adäquat) und der Anteil an imputierten Werten angegeben. Bei PISA2000 wurde nur ein zufälliges Subsample in Mathematik getestet. (Ref.): Referenzkategorien.

Quelle: TREE, eigene Berechnungen. 
Katja Scharenberg, Sandra Hupka-Brunner,

Thomas Meyer, Manfred Max Bergman (Hrsg./dir./eds)

Transitionen im Jugend-

und jungen Erwachsenenalter

Ergebnisse der Schweizer

Längsschnittstudie TREE

Transitions des adolescents

et des jeunes adultes en Suisse

Résultats de l'étude longitudinale TREE

Transitions in Youth and Young Adulthood Results from the Swiss TREE Panel Study

Volume 2

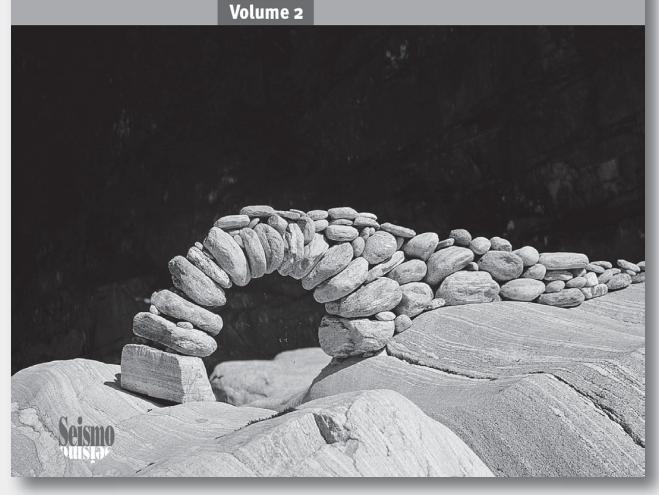

Nach dem ersten, 2011 erschienenen Band, versammelt der vorliegende zweite wiederum eine Auswahl von Beiträgen, die auf Analysen der Daten der Schweizer Längsschnittstudie TREE (Transitionen von der Erstausbildung ins Erwerbsleben) basieren. Die kritischen Übergänge im Jugend- und jungen Erwachsenenalter werden aus soziologischen, ökonomischen, psychologischen und erziehungswissenschaftlichen Blickwinkeln beleuchtet. Die Beiträge widerspiegeln damit eindrücklich das analytische und interdisziplinäre Potenzial der TREE-Daten. Thematisch steht der langfristige Einfluss der sozialen Herkunft auf Bildungs- und Erwerbsverläufe, insbesondere auf den Zugang zu höherer Bildung, im Zentrum.

Comme le premier tome publié en 2011, ce deuxième volume de l'anthologie TREE comprend une sélection d'analyses qui sont basées sur les données de l'étude longitudinale suisse TREE (Transitions de l'Ecole à l'Emploi). Les contributions présentées analysent les parcours critiques entre la fin de l'école obligatoire et l'âge adulte. Elles traitent des questions de recherche
Katja Scharenberg, Sandra Hupka-Brunner, Thomas Meyer, Manfred Max Bergman (Hrsg./dir./eds)

\section{Transitionen im Jugend- und jungen Erwachsenenalter} Ergebnisse der Schweizer Långsschnittstudie TREE

\section{Transitions des adolescents et des jeunes adultes en Suisse} Résultats de l'étude longitudinale TREE

Transitions in Youth and Young Adulthood Results from the Swiss TREE Panel Study

ISBN 978-3-03777-157-0

272 S./p., SFr. 38. - /Euro 34.-

sociologiques, économiques, psychologiques et pédagogiques, ainsi reflétant le potentiel analytique et pluri-disciplinaire des données TREE. Une des thématiques principales des contributions représentées ici est l'influence à long terme de l'origine sociale sur les parcours de formation et d'emploi, particulièrement en ce qui concerne l'accès à des formations du niveau tertiaire.

Much like the first volume published in 2011, the second volume of this anthology series gathers a selection of analyses which are empirically based on the data of the Swiss panel study TREE (Transitions from Education to Employment). The contributions of this volume carry on the investigation of the critical transitions during youth and young adulthood, drawing on sociological, economic, psychological and pedagogical research questions and thus highlighting the analytic and pluri-disciplinary research potential of the TREE data. One of the topical foci is the long-term influence of social origin on education and labour market pathways, particularly with regard to access to higher education. 